\title{
Energy Levels and the Landé $g$-Factors for Singly Ionized Lanthanum
}

\author{
Betül Karaçoban and Leyla Özdemir \\ Department of Physics, Sakarya University, 54187 Sakarya, Turkey \\ Correspondence should be addressed to Betül Karaçoban; bkaracoban@sakarya.edu.tr
}

Received 25 January 2013; Accepted 20 April 2013

Academic Editor: Gregory Lapicki

Copyright (C) 2013 B. Karaçoban and L. Özdemir. This is an open access article distributed under the Creative Commons Attribution License, which permits unrestricted use, distribution, and reproduction in any medium, provided the original work is properly cited.

We have calculated the energies and the Landé $g$-factors for $5 d^{2}, 5 d 6 s, 6 s^{2}, 4 f 6 p, 5 d 7 s, 5 d 6 d, 4 f^{2}, 6 p^{2}, 6 s 6 d, 6 s 7 s, 4 f 6 s, 4 f 5 d, 5 d 6 p$, $6 \mathrm{~s} 6 \mathrm{p}, 4 \mathrm{f} 7 \mathrm{~s}, 4 \mathrm{f} 6 \mathrm{~d}, 5 \mathrm{~d} 7 \mathrm{p}$, and $6 \mathrm{~s} 7 \mathrm{p}$ excited levels of singly ionized lanthanum (La II). These calculations have been carried out by using the multiconfiguration Hartree-Fock method within the framework of the Breit-Pauli Hamiltonian (MCHF+BP) and the relativistic Hartree-Fock (HFR) method. The obtained results have been compared with other works available in the literature. A discussion of these calculations for La II in this study has also been in view of the MCHF+BP and HFR methods.

\section{Introduction}

Lanthanides constitute a group of elements characterized by similar chemical and physical properties. The presence of this group in the periodic table is determined by behaviour of the radial part of the wave function for the $4 \mathrm{f}$ orbital, which collapses for the values of atomic number $Z>57$ and fills in the first (internal) minimum of the electrostatic potential [1]. The rare-earth element lanthanum $(Z=57)$ is a product of neutron-capture fusion reactions that occur in the late stages of stellar evolution. Lanthanum's abundance relative to other rare earths in stars of different metallicities can lead to insights on the nature of the dominant neutron-capture production sites throughout the Galaxy's history [2].

The lanthanum atom is the first member of the rareearth elements. It has two naturally occurring isotopes: ${ }^{138} \mathrm{La}$ (0.085\%) and ${ }^{139} \mathrm{La}(99.910 \%)$. Meggers viewed first the spectra of singly ionized lanthanum [3,4]. Later, Russell and Meggers analyzed the spectra of La II [5]. Grevesse and Blanquet determined the abundance of singly ionized lanthanum in the sun [6]. Spector and Gotthelf performed configuration interaction in La II [7]. Xie and coworkers investigated Rydberg and autoionization states of the singly ionized lanthanum [8]. First, ionization potential of lanthanides by laser spectroscopy was studied by Worden et al. [9]. Sugar and Reader obtained by means of a semiempirical calculation ionization potential of singly ionized lanthanum [10]. Eliav et al. reported ionization potential and excitation energies of La II [11]. Theoretical energy levels in La II were calculated by Kułaga-Egger and Migdałek [12]. In the past, different groups [13-26] investigated oscillator strengths, transition probabilities, lifetimes, and the hyperfine structure in singly ionized lanthanum by various experimental and theoretical methods. A list of energy levels for excited states was completed and presented by Sansonetti and Martin [27] and can be found on the NIST website [28].

In this work, we have presented the energies and the Landé $g$-factors for $5 \mathrm{~d}^{2}, 5 \mathrm{~d} 6 \mathrm{~s}, 6 \mathrm{~s}^{2}, 4 \mathrm{f} 6 \mathrm{p}, 5 \mathrm{~d} 7 \mathrm{~s}, 5 \mathrm{~d} 6 \mathrm{~d}, 4 \mathrm{f}^{2}, 6 \mathrm{p}^{2}$, 6s6d, 6s7s, 4f6s, 4f5d, 5d6p, 6s6p, 4f7s, 4f6d, 5d7p, and $6 s 7 p$ excited levels of singly ionized lanthanum (La II). Calculations have been carried out by the multiconfiguration Hartree-Fock method within the framework of the BreitPauli Hamiltonian $(\mathrm{MCHF}+\mathrm{BP})$ [29] and the relativistic Hartree-Fock (HFR) method [30]. These codes consider the correlation effects and relativistic corrections. These effects make an important contribution to understanding physical and chemical properties of atoms or ions, especially lanthanides. The ground-state configuration of $\mathrm{La}$ II is [Xe] $5 \mathrm{~d}^{2}{ }^{3} \mathrm{~F}_{2}$. We took into account with various configuration sets according to valence-valence and core-valence correlations for correlation effects outside the core $[\mathrm{Xe}]$ in $\mathrm{La}$ II. These configuration sets used in calculations have been 
TABLE 1: Configuration sets taken for La II in MCHF+BP and HFR calculations.

\begin{tabular}{|c|c|c|c|c|}
\hline \multirow{2}{*}{ Levels } & \multicolumn{4}{|c|}{ Configurations } \\
\hline & $\mathrm{A}$ & $\mathrm{B}$ & $\mathrm{C}$ & $\mathrm{D}$ \\
\hline \multicolumn{5}{|c|}{ For $\mathrm{MCHF}+\mathrm{BP}$ calculations } \\
\hline Even-parity & $\begin{array}{l}n \mathrm{~d}^{2}(n=5,6), 4 \mathrm{f}^{2}, 5 \mathrm{~d} 6 \mathrm{~d}, 6 \mathrm{~s} 6 \mathrm{~d}, \\
4 \mathrm{f} n \mathrm{p}, 5 \mathrm{~d} n \mathrm{~s}, n \mathrm{~s}^{2}, n \mathrm{p}^{2}(n=6-9), \\
6 \mathrm{~s} n \mathrm{~s}, 6 \mathrm{p} n \mathrm{p}, 6 \mathrm{~d} n \mathrm{~s} \\
(n=7-9), 7 \mathrm{~s} n \mathrm{~s}, 7 \mathrm{p} n \mathrm{p}(n=8, \\
9), 8 \mathrm{~s} 9 \mathrm{~s}, 8 \mathrm{p} 9 \mathrm{p}\end{array}$ & $\begin{array}{l}\text { As in } \\
\text { calculation A }\end{array}$ & $\begin{array}{l}5 p^{6} 5 d^{2}, 5 p^{6} 5 d 6 s \\
5 p^{6} 6 s^{2}, 5 p^{6} 4 f 6 p \\
5 p^{6} 4 f^{2}, 5 p^{5} 4 f 6 s^{2} \\
5 p^{5} 5 d^{2} 6 p, 5 p^{5} 6 s^{2} 6 p\end{array}$ & $\begin{array}{l}5 \mathrm{p}^{6} n \mathrm{~d}^{2}(n=5,6), 5 \mathrm{p}^{6} 4 \mathrm{f}^{2}, \\
5 \mathrm{p}^{6} 5 \mathrm{~d} 6 \mathrm{~d}, 5 \mathrm{p}^{6} 6 \mathrm{~s} 6 \mathrm{~d}, 5 \mathrm{p}^{6} 4 \mathrm{f} n \mathrm{p}, \\
5 \mathrm{p}^{6} 5 \mathrm{~d} n \mathrm{~s}, 5 \mathrm{p}^{6} n \mathrm{~s}^{2}, 5 \mathrm{p}^{6} n \mathrm{p}^{2}(n \\
=6-9), 5 \mathrm{p}^{6} 6 \mathrm{~s} n \mathrm{~s}, 5 \mathrm{p}^{6} 6 \mathrm{~d} n \mathrm{~s}, \\
5 \mathrm{p}^{6} 6 \mathrm{p} n \mathrm{p}(n=7-9), 5 \mathrm{p}^{6} 7 \mathrm{~s} n \mathrm{~s} \\
(n=8,9), 5 \mathrm{p}^{6} 7 \mathrm{p} 8 \mathrm{p}, \\
5 \mathrm{p}^{6} 8 \mathrm{~s} 9 \mathrm{~s}, 5 \mathrm{p}^{6} 8 \mathrm{p} 9 \mathrm{p}\end{array}$ \\
\hline Odd-parity & $\begin{array}{l}4 \mathrm{f} 6 \mathrm{~s}, 4 \mathrm{f} 5 \mathrm{~d}, 5 \mathrm{~d} n \mathrm{p}, 6 \mathrm{~s} n \mathrm{p}(n= \\
6-9), 6 \mathrm{p} n \mathrm{~s}, 7 \mathrm{~s} n \mathrm{p} \\
(n=7-9), 7 \mathrm{p} n \mathrm{~s}, 8 \mathrm{~s} n \mathrm{p}(n=8 \\
9), 8 \mathrm{p} 9 \mathrm{~s}, 9 \mathrm{~s} 9 \mathrm{p}\end{array}$ & $\begin{array}{l}\text { As in } \\
\text { calculation A }\end{array}$ & $\begin{array}{l}5 p^{6} 4 f 6 s, 5 p^{6} 4 f 5 d, 5 p^{6} 5 d 6 p \\
5 p^{5} 4 f^{2} 6 s \\
5 p^{5} 5 d 6 s^{2}, 5 p^{5} 5 d 6 p^{2}\end{array}$ & \\
\hline \multicolumn{5}{|c|}{ For HFR calculations } \\
\hline Even-parity & $5 d^{2}, 5 d 6 s, 6 s^{2}, 4 f 6 p, 4 f^{2}$ & $\begin{array}{l}5 \mathrm{~d}^{2}, 5 \mathrm{~d} 6 \mathrm{~s}, 6 \mathrm{~s}^{2}, \\
4 \mathrm{f} 6 \mathrm{p}, 5 \mathrm{~d} 7 \mathrm{~s}, \\
5 \mathrm{~d} 6 \mathrm{~d}, 4 \mathrm{f}^{2}, 6 \mathrm{p}^{2}, \\
6 \mathrm{~s} 6 \mathrm{~d}, 6 \mathrm{~s} 7 \mathrm{~s}\end{array}$ & & \\
\hline Odd-parity & $4 f 6 s, 4 f 5 d, 5 d 6 p, 6 s 6 p$ & $\begin{array}{l}4 f 6 s, 4 f 5 d, 5 d 6 \\
6 s 6 p, 4 f 7 s, 4 f 6 \\
5 d 7 p, 6 s 7 p\end{array}$ & & \\
\hline
\end{tabular}

denoted by A, B, C, and D for the $\mathrm{MCHF}+\mathrm{BP}$, and A and B for the HFR calculations and given in Table 1 . We have performed the atomic structure calculations on lanthanide atoms and ions, systematically. We reported some works related to these atoms and ions using the methods mentioned above [3142]. In addition, we presented the energies of $5 \mathrm{~d}^{2}, 5 \mathrm{~d} 6 \mathrm{~s}, 6 \mathrm{~s}^{2}$, and $6 \mathrm{p}^{2}$ excited levels and ionization energy for La II by the MCHF+BP method [34]. In this work, we have considered more levels than in [34] and added the Landé $g$-factors for these levels.

\section{Calculation Methods: MCHF and HFR}

In the MCHF method [29], atomic state functions can be obtained as a linear combination of configuration state functions (CSFs) in LS coupling,

$$
\Psi(\gamma L S)=\sum_{i=1}^{M} c_{i} \Phi\left(\gamma_{i} L S\right), \quad \sum_{i=1}^{M} c_{i}^{2}=1 .
$$

The mixing coefficients $\left\{c_{i}\right\}$ and the radial orbitals are optimized simultaneously, based on the expectation values $\langle\Psi|H| \Psi\rangle$.

In the MCHF method, the Breit-Pauli Hamiltonian for relativistic corrections is taken as a perturbation with order $\alpha^{2}$. The Breit-Pauli Hamiltonian includes relativistic effects. This Hamiltonian can be written as follows:

$$
H_{\mathrm{BP}}=H_{\mathrm{NR}}+H_{\mathrm{RS}}+H_{\mathrm{FS}} \text {, }
$$

where $H_{\mathrm{NR}}$ is the nonrelativistic many-electron Hamiltonian and $H_{\mathrm{RS}}$ is the relativistic shift operator including mass correction, one- and two-body Darwin terms, spin-spin contact term, and orbit-orbit term; fine structure Hamiltonian
$\mathrm{H}_{\mathrm{FS}}$ consists of the spin-orbit, spin-other-orbit, and spinspin terms. Now, the multiconfiguration wave functions are obtained as linear combinations of CSFs in LSJ coupling. Therefore, the radial functions building the CSFs are taken from a previous nonrelativistic MCHF run and only the expansion coefficients are optimized. Therefore, the matrix eigenvalue problem becomes

$$
\mathbf{H} c=E c,
$$

where $\mathbf{H}$ is the Hamiltonian matrix with the following elements

$$
H_{i j}=\left\langle\gamma_{i} L_{i} S_{i} J M\left|H_{\mathrm{BP}}\right| \gamma_{j} L_{j} S_{j} J M\right\rangle .
$$

The Breit-Pauli Hamiltonian is a first order perturbation correction to the nonrelativistic Hamiltonian. The Landé $g$ factor of an atomic level is related to the energy shift of the sublevels having magnetic number $M$ by

$$
\Delta E(\gamma L S)=\mu_{B} B g_{\gamma J} M,
$$

where $B$ is the magnetic field intensity and $\mu_{B}$ is the Bohr magneton. In pure $L S$ coupling, the Landé $g$-factor can be taken given by formula (8) in [43]. The Landé $g$-factors for energy levels are a valuable aid in the analysis of a spectrum. These factors which are a measure of the magnetic sensitivity of atomic levels can be calculated using the code developed by Jönsson and Gustafsson [43] according to MCHF wave functions.

In the HFR method [30], for $N$ electron atom of nuclear charge $Z_{0}$, the Hamiltonian is expanded as

$$
\mathbf{H}=-\sum_{i} \nabla_{i}^{2}-\sum_{i} \frac{2 Z_{0}}{r_{i}}+\sum_{i>j} \frac{2}{r_{i j}}+\sum_{i} \zeta_{i}\left(r_{i}\right) \mathbf{l}_{i} \cdot \mathbf{s}_{i}
$$


in atomic units with the distance $r_{i}$ of the $i$ th electron from the nucleus and $r_{i j}=\left|\mathbf{r}_{i}-\mathbf{r}_{j}\right| . \zeta_{i}(R)=\left(\alpha^{2} / 2\right)(1 / r)(\partial V / \partial r)$ is the spin-orbit term with $\alpha$ the fine structure constant and $V$ the mean potential field due to the nucleus and other electrons.

Wave function $|\gamma J M\rangle$ of the $M$ sublevel of a level labeled $\gamma J$ is expressed in terms of $L S$ basis states $|\alpha L S J M\rangle$ by the following formula:

$$
|\gamma J M\rangle=\sum_{\alpha L S}|\alpha L S J M\rangle\langle\alpha L S J \mid \gamma J\rangle .
$$

Using determinant wave functions for the atom, total binding energy is given by

$$
E=\sum_{i}\left(E_{k}^{i}+E_{n}^{i}+\sum_{j<i} E^{i j}\right),
$$

where $E_{k}^{i}$ is the kinetic energy, $E_{n}^{i}$ is the electron-nuclear energy, and $E_{i j}$ is the Coulomb interaction energy between electron $i$ and $j$, averaged over all possible magnetic quantum numbers.

This method calculates one-electron radial wave functions for each of any number of specified electron configurations, using the Hartree-Fock or any of several more approximate methods. It obtains the center-of-gravity energy of each configuration and those radial Coulomb and spin-orbit integrals required to calculate the energy levels for the configuration. After the wave functions have been obtained, they are used to calculate the configuration-interaction Coulomb integrals between each pair of interacting configurations. Then, energy matrices are set up for each possible value of $J$, and each matrix is diagonalized to get eigenvalues (energy levels) and eigenvectors (multiconfiguration, intermediate coupling wave functions in various possible angularmomentum coupling representations).

Relativistic corrections to total binding energies become quite large for heavy elements; the main contributions come from the tightly bound inner electrons. In the HFR method there have been limited calculations to the mass-velocity and Darwin corrections by using relativistic correction to total binding energy:

$$
E_{r}=\sum_{i} E_{r}^{i}=\sum_{i}\left(E_{m}^{i}+E_{D}^{i}\right)
$$

The Landé $g$-factors in HFR calculations have the same formula as in MCHF calculations. Here, the calculations for the Landé $g$-factors have been performed according to HFR wave functions.

\section{Results and Discussion}

Here, we have calculated the energies and the Landé $g$-factors for $5 \mathrm{~d}^{2}, 5 \mathrm{~d} 6 \mathrm{~s}, 6 \mathrm{~s}^{2}, 4 \mathrm{f} 6 \mathrm{p}, 5 \mathrm{~d} 7 \mathrm{~s}, 5 \mathrm{~d} 6 \mathrm{~d}, 4 \mathrm{f}^{2}, 6 \mathrm{p}^{2}, 6 \mathrm{~s} 6 \mathrm{~d}, 6 \mathrm{~s} 7 \mathrm{~s}$, 4f6s, 4f5d, 5d6p, 6s6p, 4f7s, 4f6d, 5d7p, and 6s7p excited levels outside the core [Xe] in La II using the MCHF+BP [44] and HFR [45] codes. We have presented the several excited level energies and transition energies for La I and La II [34]. We have performed new various calculations for obtaining configuration state functions (CSFs) according to valence-valence and core-valence correlations. Moreover, it is well known that the Landé $g$-factors are important in many scientific areas such as astrophysics. Table 1 displays the various configuration sets for considering correlation effects. The results for energy levels and the Landé $g$-factors of La II have been reported in Table 2. In this table, the calculations for the various configuration sets are represented by $\mathrm{A}, \mathrm{B}, \mathrm{C}$, and $\mathrm{D}$ for the $\mathrm{MCHF}+\mathrm{BP}$ and by $\mathrm{A}$ and $\mathrm{B}$ for the HFR calculations. A comparison is also made with other calculations and experiments in the table. References for other comparison values are typed below the table with a superscript lowercase letter. Only odd-parity states in table are indicated by the superscript "o."

Electron correlation effects and relativistic effects play an important role in the spectra of heavy elements. Thus, we have to consider these effects for lanthanides. However, it is very difficult to calculate the electron correlation for these atoms because of their complex structures. Although this provides useful information for understanding the correlation effect, computer constraints occur. Therefore, we increasingly varied some parameters in the MCHF atomic structure package (maximum number of eigenpairs, maximum number of configuration state functions, maximum number of terms, and maximum number of coefficients) so that the calculations for the configurations above could reasonably be made. The energies and the Landé $g$-factors for $5 d^{2}, 5 d 6 s, 6 s^{2}, 4 f 6 p$, $5 \mathrm{~d} 7 \mathrm{~s}, 5 \mathrm{~d} 6 \mathrm{~d}, 4 \mathrm{f}^{2}, 6 \mathrm{p}^{2}, 6 \mathrm{~s} 6 \mathrm{~d}, 6 \mathrm{~s} 7 \mathrm{~s}, 4 \mathrm{f} 6 \mathrm{~s}, 4 \mathrm{f} 5 \mathrm{~d}, 5 \mathrm{~d} 6 \mathrm{p}, 6 \mathrm{~s} 6 \mathrm{p}, 4 \mathrm{f} 7 \mathrm{~s}$, $4 \mathrm{f} 6 \mathrm{~d}, 5 \mathrm{~d} 7 \mathrm{p}$, and $6 \mathrm{~s} 7 \mathrm{p}$ excited levels outside the core [Xe] in La II are calculated by the MCHF+BP and HFR methods. The obtained results have been presented as energies $\left(\mathrm{cm}^{-1}\right)$ relative to $5 \mathrm{~d}^{2}{ }^{3} \mathrm{~F}_{2}$ ground state in Table 2 .

In the $\mathrm{MCHF}+\mathrm{BP}$ calculations, it is taken into account core [Xe] for calculations $A$ and $B$ and core [Cd] for calculations C and D for La II. The odd- and even-parity levels of calculations A, B, and D are considered the only interaction between the valence electrons, whereas odd- and even-parity levels of calculation $\mathrm{C}$ were an included interaction between valence-valence electrons and core-valence electrons. In the MCHF+BP method, firstly, for configurations selected according to valence-valence and core-valence correlations, the non-relativistic wave functions and energies were obtained. In the configuration interaction method, energy levels are performed using wave functions obtained taking into account relativistic corrections. Then the Landé $g$-factors for energy levels are calculated using the Zeeman program developed by Jönsson and Gustafsson [43].

In the $\mathrm{MCHF}+\mathrm{BP}$ calculations, we have reported that the levels belong to $5 d^{2}, 5 d 6 s, 6 s^{2}, 5 d 6 d, 6 p^{2}$, and $6 s 6 d$ for evenparity and 4f6s, 4f5d, 5d6p, and 6s6p for odd-parity in Table 2. We presented the energies of several even-parity levels from obtained calculation A in [34]. Although configurations of calculation B are the same with calculations of calculation A, $\mathrm{MCHF}$ run is different. When our $\mathrm{MCHF}+\mathrm{BP}$ results were compared with others $[12,28]$, the energies for most of levels are in agreement. $5 \mathrm{~d}^{2}{ }^{3} \mathrm{~F}$ levels obtained from calculations $\mathrm{A}$ and $\mathrm{D}$ and $5 \mathrm{~d}^{2}{ }^{3} \mathrm{P}$ levels obtained from calculations $\mathrm{A}$ and $\mathrm{C}$ are good in agreement with other works. The agreement is 
TABLE 2: Energies, $E$, and the Landé $g$-factors for some levels in La II.

\begin{tabular}{|c|c|c|c|c|c|c|c|}
\hline \multicolumn{2}{|c|}{ Levels } & \multicolumn{3}{|c|}{$E\left(\mathrm{~cm}^{-1}\right)$} & \multicolumn{3}{|c|}{$g$-Factors } \\
\hline \multirow{2}{*}{ Conf. } & \multirow{2}{*}{ Term } & \multicolumn{2}{|c|}{ This work } & \multirow{2}{*}{ Other works } & \multicolumn{2}{|c|}{ This work } & \multirow{2}{*}{ Other works } \\
\hline & & $\mathrm{MCHF}+\mathrm{BP}$ & HFR & & $\mathrm{MCHF}+\mathrm{BP}$ & HFR & \\
\hline \multicolumn{8}{|c|}{ For even-parity } \\
\hline \multirow[t]{12}{*}{$5 d^{2}$} & ${ }^{3} \mathrm{~F}_{2}$ & $0.00^{A, B, C, D}$ & $-0.13^{\mathrm{A}}$ & $0.00^{\mathrm{a}}$ & $0.728^{\mathrm{A}}$ & $0.732^{\mathrm{A}}$ & $0.721^{\mathrm{a}}$ \\
\hline & & & $0.02^{\mathrm{B}}$ & $-4^{\mathrm{b}}$ & $0.709^{\mathrm{B}}$ & $0.736^{\mathrm{B}}$ & \\
\hline & & & & & $0.715^{\mathrm{C}}$ & & \\
\hline & & & & & $0.722^{\mathrm{D}}$ & & \\
\hline & ${ }^{3} \mathrm{~F}_{3}$ & $1100.72^{\mathrm{A}}$ & $1018.92^{\mathrm{A}}$ & $1016.100^{\mathrm{a}}$ & $1.083^{\mathrm{A}, \mathrm{B}, \mathrm{C}, \mathrm{D}}$ & $1.084^{\mathrm{A}, \mathrm{B}}$ & $1.038^{\mathrm{a}}$ \\
\hline & & $816.18^{\mathrm{B}}$ & $1046.86^{\mathrm{B}}$ & $1028^{\mathrm{b}}$ & & & \\
\hline & & $743.03^{C}$ & & & & & \\
\hline & & $898.90^{\mathrm{D}}$ & & & & & \\
\hline & ${ }^{3} \mathrm{~F}_{4}$ & $2131.38^{\mathrm{A}}$ & $1935.49^{\mathrm{A}}$ & $1970.700^{\mathrm{a}}$ & $1.249^{\mathrm{A}, \mathrm{B}, \mathrm{C}}$ & $1.249^{\mathrm{A}, \mathrm{B}}$ & $1.248^{\mathrm{a}}$ \\
\hline & & $1612.45^{\mathrm{B}}$ & $1974.01^{\mathrm{B}}$ & $1963^{b}$ & $1.250^{\mathrm{D}}$ & & \\
\hline & & $1456.54^{\mathrm{C}}$ & & & & & \\
\hline & & $1741.31^{\mathrm{D}}$ & & & & & \\
\hline \multirow[t]{4}{*}{$5 d^{2}$} & ${ }^{1} \mathrm{D}_{2}$ & $1379.80^{\mathrm{A}}$ & $1394.91^{\mathrm{A}}$ & $1394.460^{\mathrm{a}}$ & $0.993^{\mathrm{A}}$ & $0.965^{\mathrm{A}}$ & $0.977^{\mathrm{a}}$ \\
\hline & & $1443.25^{\mathrm{B}}$ & $1393.79^{\mathrm{B}}$ & $1395^{\mathrm{b}}$ & $0.968^{B, C}$ & $0.959^{\mathrm{B}}$ & \\
\hline & & $1236.03^{\mathrm{C}}$ & & & $0.955^{\mathrm{D}}$ & & \\
\hline & & $1398.12^{\mathrm{D}}$ & & & & & \\
\hline \multirow[t]{12}{*}{$5 d^{2}$} & ${ }^{3} \mathrm{P}_{0}$ & $5377.78^{\mathrm{A}}$ & $5228.71^{\mathrm{A}}$ & $5249.700^{\mathrm{a}}$ & & & \\
\hline & & $5918.77^{\mathrm{B}}$ & $5265.77^{\mathrm{B}}$ & $5244^{\mathrm{b}}$ & & & \\
\hline & & $5575.39^{\mathrm{C}}$ & & & & & \\
\hline & & $5654.01^{\mathrm{D}}$ & & & & & \\
\hline & ${ }^{3} \mathrm{P}_{1}$ & $6000.10^{\mathrm{A}}$ & $5769.81^{\mathrm{A}}$ & $5718.120^{\mathrm{a}}$ & $1.501^{\mathrm{A}, \mathrm{B}, \mathrm{C}, \mathrm{D}}$ & $1.501^{\mathrm{A}, \mathrm{B}}$ & $1.497^{\mathrm{a}}$ \\
\hline & & $6374.80^{\mathrm{B}}$ & $5737.42^{\mathrm{B}}$ & $5725^{\mathrm{b}}$ & & & \\
\hline & & $5867.74^{\mathrm{C}}$ & & & & & \\
\hline & & $6132.57^{\mathrm{D}}$ & & & & & \\
\hline & ${ }^{3} \mathrm{P}_{2}$ & $6513.01^{\mathrm{A}}$ & $6261.60^{\mathrm{A}}$ & $6227.420^{\mathrm{a}}$ & $1.485^{\mathrm{A}}$ & $1.489^{\mathrm{A}}$ & $1.481^{\mathrm{a}}$ \\
\hline & & $6798.78^{\mathrm{B}}$ & $6239.70^{\mathrm{B}}$ & $6224^{\mathrm{b}}$ & $1.494^{\mathrm{B}, \mathrm{D}}$ & $1.488^{\mathrm{B}}$ & \\
\hline & & $6247.36^{\mathrm{C}}$ & & & $1.497^{\mathrm{C}}$ & & \\
\hline & & $6588.07^{\mathrm{D}}$ & & & & & \\
\hline \multirow[t]{4}{*}{$5 d^{2}$} & ${ }^{1} \mathrm{G}_{4}$ & $8739.05^{\mathrm{A}}$ & $7445.09^{\mathrm{A}}$ & $7473.320^{\mathrm{a}}$ & $1.002^{\mathrm{A}}$ & $1.002^{\mathrm{A}, \mathrm{B}}$ & $1.000^{\mathrm{a}}$ \\
\hline & & $8468.71^{\mathrm{B}}$ & $7374.70^{\mathrm{B}}$ & $7476^{\mathrm{b}}$ & $1.001^{\mathrm{B}, \mathrm{C}, \mathrm{D}}$ & & \\
\hline & & $8941.04^{\mathrm{C}}$ & & & & & \\
\hline & & $9027.98^{\mathrm{D}}$ & & & & & \\
\hline \multirow[t]{3}{*}{$5 d^{2}$} & ${ }^{1} \mathrm{~S}_{0}$ & $16629.75^{\mathrm{A}}$ & $16453.30^{\mathrm{A}}$ & - & & & \\
\hline & & $18979.63^{\mathrm{B}}$ & $13675.30^{\mathrm{B}}$ & & & & \\
\hline & & $18143.98^{\mathrm{D}}$ & & & & & \\
\hline \multirow[t]{12}{*}{$5 \mathrm{~d} 6 \mathrm{~s}$} & ${ }^{3} \mathrm{D}_{1}$ & $1662.59^{A}$ & $1893.01^{\mathrm{A}}$ & $1895.150^{\mathrm{a}}$ & $0.499^{\mathrm{A}, \mathrm{B}, \mathrm{C}, \mathrm{D}}$ & $0.499^{\mathrm{A}, \mathrm{B}}$ & $0.498^{\mathrm{a}}$ \\
\hline & & $2481.69^{\mathrm{B}}$ & $1898.19^{\mathrm{B}}$ & $1902^{\mathrm{b}}$ & & & \\
\hline & & $1994.95^{\mathrm{C}}$ & & & & & \\
\hline & & $2533.26^{\mathrm{D}}$ & & & & & \\
\hline & ${ }^{3} \mathrm{D}_{2}$ & $2476.73^{\mathrm{A}}$ & $2580.06^{\mathrm{A}}$ & $2591.600^{\mathrm{a}}$ & $1.119^{\mathrm{A}}$ & $1.143^{\mathrm{A}}$ & $1.133^{\mathrm{a}}$ \\
\hline & & $2939.18^{\text {B }}$ & $2549.72^{\mathrm{B}}$ & $2572^{\mathrm{b}}$ & $1.159^{\mathrm{B}}$ & $1.144^{\mathrm{B}}$ & \\
\hline & & $2550.88^{C}$ & & & $1.154^{\mathrm{C}}$ & & \\
\hline & & $3010.41^{\mathrm{D}}$ & & & $1.160^{\mathrm{D}}$ & & \\
\hline & ${ }^{3} \mathrm{D}_{3}$ & $3094.79^{\mathrm{A}}$ & $3311.82^{\mathrm{A}}$ & $3250.350^{\mathrm{a}}$ & $1.334^{\mathrm{A}, \mathrm{B}, \mathrm{C}, \mathrm{D}}$ & $1.334^{\mathrm{A}, \mathrm{B}}$ & $1.334^{\mathrm{a}}$ \\
\hline & & $3501.39^{\mathrm{B}}$ & $3255.89^{\mathrm{B}}$ & $3260^{\mathrm{b}}$ & & & \\
\hline & & $3204.83^{C}$ & & & & & \\
\hline & & $3614.27^{\mathrm{D}}$ & & & & & \\
\hline $5 \mathrm{~d} 6 \mathrm{~s}$ & ${ }^{1} \mathrm{D}_{2}$ & $10064.87^{\mathrm{A}}$ & $10088.91^{\mathrm{A}}$ & $10094.800^{\mathrm{a}} ?$ & $1.010^{\mathrm{A}}$ & $1.006^{\mathrm{A}, \mathrm{B}}$ & $1.005^{\mathrm{a}}$ \\
\hline
\end{tabular}


TABLE 2: Continued.

\begin{tabular}{|c|c|c|c|c|c|c|c|}
\hline \multicolumn{2}{|c|}{ Levels } & \multicolumn{3}{|c|}{$E\left(\mathrm{~cm}^{-1}\right)$} & \multicolumn{3}{|c|}{$g$-Factors } \\
\hline \multirow{2}{*}{ Conf. } & \multirow{2}{*}{ Term } & \multicolumn{2}{|c|}{ This work } & \multirow{2}{*}{ Other works } & \multicolumn{2}{|c|}{ This work } & \multirow{2}{*}{ Other works } \\
\hline & & $\mathrm{MCHF}+\mathrm{BP}$ & HFR & & MCHF+BP & HFR & \\
\hline & & $11480.98^{\mathrm{B}}$ & $10095.00^{\mathrm{B}}$ & $10096^{\mathrm{b}}$ & $1.003^{\mathrm{B}, \mathrm{D}}$ & & \\
\hline & & $13078.05^{\mathrm{C}}$ & & & $1.001^{\mathrm{C}}$ & & \\
\hline & & $11980.57^{\mathrm{D}}$ & & & & & \\
\hline \multirow[t]{4}{*}{$6 s^{2}$} & ${ }^{1} S_{0}$ & $6428.50^{\mathrm{A}}$ & $7371.89^{A}$ & $7394.570^{\mathrm{a}}$ & & & \\
\hline & & $6664.52^{\mathrm{B}}$ & $7393.30^{\mathrm{B}}$ & $7395^{\mathrm{b}}$ & & & \\
\hline & & $9713.71^{\mathrm{C}}$ & & & & & \\
\hline & & $6709.28^{\mathrm{D}}$ & & & & & \\
\hline \multirow[t]{6}{*}{$4 f 6 p$} & ${ }^{3} \mathrm{~F}_{2}$ & - & $35544.74^{\mathrm{A}}$ & $35787.53^{\mathrm{a}}$ & - & $0.669^{\mathrm{A}}$ & $0.719^{\mathrm{a}}$ \\
\hline & & & $35756.53^{B}$ & $35771^{b}$ & & $0.747^{\mathrm{B}}$ & \\
\hline & ${ }^{3} \mathrm{~F}_{3}$ & - & $35687.88^{\mathrm{A}}$ & $36954.65^{\mathrm{a}}$ & - & $1.050^{\mathrm{A}}$ & $1.061^{\mathrm{a}}$ \\
\hline & & & $37062.30^{\text {B }}$ & $36953^{\mathrm{b}}$ & & $1.005^{\mathrm{B}}$ & \\
\hline & ${ }^{3} \mathrm{~F}_{4}$ & - & $37018.59^{\mathrm{A}}$ & $37790.57^{\mathrm{a}}$ & - & $1.212^{\mathrm{A}}$ & $1.113^{\mathrm{a}}$ \\
\hline & & & $37733.47^{\mathrm{B}}$ & $37779^{\mathrm{b}}$ & & $1.105^{\mathrm{B}}$ & \\
\hline \multirow[t]{2}{*}{$4 f 6 p$} & ${ }^{1} \mathrm{~F}_{3}$ & - & $36917.97^{\mathrm{A}}$ & $37209.71^{a}$ & - & $0.934^{\mathrm{A}}$ & $0.944^{\mathrm{a}}$ \\
\hline & & & $37302.79^{\mathrm{B}}$ & $37243^{\mathrm{b}}$ & & $1.036^{\mathrm{B}}$ & \\
\hline \multirow[t]{6}{*}{$4 f 6 p$} & ${ }^{3} \mathrm{G}_{3}$ & - & $37604.82^{\mathrm{A}}$ & $35452.66^{\mathrm{a}}$ & - & $0.854^{\mathrm{A}}$ & $0.876^{\mathrm{a}}$ \\
\hline & & & $35373.81^{\mathrm{B}}$ & $35465^{\mathrm{b}}$ & & $0.856^{\mathrm{B}}$ & \\
\hline & ${ }^{3} \mathrm{G}_{4}$ & - & $37768.59^{A}$ & $37172.79^{\mathrm{a}}$ & - & $1.068^{\mathrm{A}}$ & $1.127^{\mathrm{a}}$ \\
\hline & & & $37186.27^{\mathrm{B}}$ & $37157^{\mathrm{b}}$ & & $1.098^{\mathrm{B}}$ & \\
\hline & ${ }^{3} \mathrm{G}_{5}$ & - & $39201.02^{\mathrm{A}}$ & $39018.74^{\mathrm{a}}$ & - & $1.200^{\mathrm{A}, \mathrm{B}}$ & $1.21^{\mathrm{a}}$ \\
\hline & & & $39035.52^{\mathrm{B}}$ & $39007^{\mathrm{b}}$ & & & \\
\hline \multirow[t]{6}{*}{$4 f 6 p$} & ${ }^{3} \mathrm{D}_{1}$ & - & $38123.37^{\mathrm{A}}$ & $38534.11^{\mathrm{a}}$ & - & $0.499^{\mathrm{A}, \mathrm{B}}$ & $0.497^{\mathrm{a}}$ \\
\hline & & & $38536.02^{\mathrm{B}}$ & $38545^{\mathrm{b}}$ & & & \\
\hline & ${ }^{3} \mathrm{D}_{2}$ & - & $38214.22^{\mathrm{A}}$ & $38221.49^{\mathrm{a}}$ & - & $1.131^{\mathrm{A}}$ & $1.071^{\mathrm{a}}$ \\
\hline & & & $38070.87^{\mathrm{B}}$ & $38210^{\mathrm{b}}$ & & $1.027^{\mathrm{B}}$ & \\
\hline & ${ }^{3} \mathrm{D}_{3}$ & - & $39512.21^{A}$ & $39402.55^{\mathrm{a}}$ & - & $1.329^{\mathrm{A}}$ & $1.274^{\mathrm{a}}$ \\
\hline & & & $39535.50^{\mathrm{B}}$ & $39403^{\mathrm{b}}$ & & $1.270^{\mathrm{B}}$ & \\
\hline \multirow[t]{2}{*}{$4 \mathrm{f} 6 \mathrm{p}$} & ${ }^{1} \mathrm{G}_{4}$ & - & $38968.77^{\mathrm{A}}$ & $39221.65^{\mathrm{a}}$ & - & $1.021^{\mathrm{A}}$ & $1.059^{\mathrm{a}}$ \\
\hline & & & $39162.52^{\mathrm{B}}$ & $39235^{\mathrm{b}}$ & & $1.097^{\mathrm{B}}$ & \\
\hline \multirow[t]{2}{*}{$4 f 6 p$} & ${ }^{1} \mathrm{D}_{2}$ & - & $40343.10^{\mathrm{A}}$ & $40457.71^{\mathrm{a}}$ & - & $1.033^{\mathrm{A}}$ & $1.036^{\mathrm{a}}$ \\
\hline & & & $40233.91^{\mathrm{B}}$ & $40456^{\mathrm{b}}$ & & $1.059^{\mathrm{B}}$ & \\
\hline \multirow[t]{5}{*}{$5 \mathrm{~d} 7 \mathrm{~s}$} & ${ }^{3} \mathrm{D}_{1}$ & - & $49703.78^{\mathrm{B}}$ & $49733.13^{\mathrm{a}}$ & - & $0.500^{\mathrm{B}}$ & $0.500^{\mathrm{a}}$ \\
\hline & & & & $49714^{\mathrm{b}}$ & & & \\
\hline & ${ }^{3} \mathrm{D}_{2}$ & - & $49952.52^{\mathrm{B}}$ & $49884.35^{\mathrm{a}}$ & - & $1.128^{\mathrm{B}}$ & $1.117^{\mathrm{a}}$ \\
\hline & & & & $49905^{\mathrm{b}}$ & & & \\
\hline & ${ }^{3} \mathrm{D}_{3}$ & - & $51238.10^{\mathrm{B}}$ & $51228.57^{\mathrm{a}}$ & - & $1.307^{\mathrm{B}}$ & $1.315^{\mathrm{a}}$ \\
\hline $5 \mathrm{~d} 7 \mathrm{~s}$ & ${ }^{1} \mathrm{D}_{2}$ & - & $51501.10^{\mathrm{B}}$ & $51523.86^{\mathrm{a}}$ & - & $1.058^{\mathrm{B}}$ & $1.036^{\mathrm{a}}$ \\
\hline & & & & $51516^{\mathrm{b}}$ & & & \\
\hline \multirow[t]{2}{*}{$5 \mathrm{~d} 6 \mathrm{~d}$} & ${ }^{1} \mathrm{~F}_{3}$ & $70728.01^{\mathrm{A}}$ & $51978.02^{\mathrm{B}}$ & $52137.67^{\mathrm{a}}$ & $0.978^{A}$ & $1.048^{\mathrm{B}}$ & $0.987^{\mathrm{a}}$ \\
\hline & & $74919.76^{\mathrm{B}}$ & & $52216^{\mathrm{b}}$ & $1.056^{\mathrm{B}}$ & & \\
\hline \multirow[t]{6}{*}{$5 \mathrm{~d} 6 \mathrm{~d}$} & ${ }^{3} \mathrm{D}_{1}$ & $71108.98^{\mathrm{A}}$ & $52220.02^{\mathrm{B}}$ & $52169.66^{\mathrm{a}}$ & $0.544^{\mathrm{A}}$ & $0.557^{\mathrm{B}}$ & $0.621^{\mathrm{a}}$ \\
\hline & & $75022.05^{\mathrm{B}}$ & & $52148^{\mathrm{b}}$ & $0.622^{\mathrm{B}}$ & & \\
\hline & ${ }^{3} \mathrm{D}_{2}$ & $71922.34^{\mathrm{A}}$ & $52746.60^{\mathrm{B}}$ & $52734.81^{\mathrm{a}}$ & $1.167^{\mathrm{A}}$ & $1.126^{\mathrm{B}}$ & $1.154^{\mathrm{a}}$ \\
\hline & & $75364.62^{\mathrm{B}}$ & & $52728^{\mathrm{b}}$ & $1.033^{\mathrm{B}}$ & & \\
\hline & ${ }^{3} \mathrm{D}_{3}$ & $72905.11^{\mathrm{A}}$ & $53276.41^{\mathrm{B}}$ & $53689.56^{\mathrm{a}}$ & $1.302^{\mathrm{A}}$ & $1.297^{\mathrm{B}}$ & $1.218^{\mathrm{a}}$ \\
\hline & & $75927.05^{\mathrm{B}}$ & & $53647^{\mathrm{b}}$ & $1.119^{\mathrm{B}}$ & & \\
\hline $5 \mathrm{~d} 6 \mathrm{~d}$ & ${ }^{1} \mathrm{P}_{1}$ & $74454.96^{\mathrm{A}}$ & $53356.36^{\mathrm{B}}$ & $53302.56^{\mathrm{a}}$ & $1.048^{\mathrm{A}}$ & $1.343^{\mathrm{B}}$ & $1.335^{\mathrm{a}}$ \\
\hline
\end{tabular}


TABLE 2: Continued.

\begin{tabular}{|c|c|c|c|c|c|c|c|}
\hline \multicolumn{2}{|c|}{ Levels } & \multicolumn{3}{|c|}{$E\left(\mathrm{~cm}^{-1}\right)$} & \multicolumn{3}{|c|}{$g$-Factors } \\
\hline \multirow{2}{*}{ Conf. } & \multirow{2}{*}{ Term } & \multicolumn{2}{|c|}{ This work } & \multirow{2}{*}{ Other works } & \multicolumn{2}{|c|}{ This work } & \multirow{2}{*}{ Other works } \\
\hline & & $\mathrm{MCHF}+\mathrm{BP}$ & HFR & & $\mathrm{MCHF}+\mathrm{BP}$ & HFR & \\
\hline \multirow{7}{*}{$5 \mathrm{~d} 6 \mathrm{~d}$} & \multirow{3}{*}{${ }^{3} \mathrm{~F}_{2}$} & $75644.43^{\mathrm{B}}$ & & $53317^{\mathrm{b}}$ & $1.373^{\mathrm{B}}$ & \multirow{3}{*}{$0.741^{\mathrm{B}}$} & \multirow{3}{*}{$0.751^{\mathrm{a}}$} \\
\hline & & $86857.66^{\mathrm{A}}$ & \multirow[t]{2}{*}{$53855.01^{\mathrm{B}}$} & $53885.24^{\mathrm{a}}$ & $0.687^{\mathrm{A}}$ & & \\
\hline & & $75086.62^{\mathrm{B}}$ & & $53914^{\mathrm{b}}$ & $0.847^{\mathrm{B}}$ & & \\
\hline & \multirow[t]{2}{*}{${ }^{3} \mathrm{~F}_{3}$} & $76042.84^{\mathrm{B}}$ & \multirow[t]{2}{*}{$54392.59^{\text {B }}$} & $54840.04^{\mathrm{a}}$ & $1.161^{\mathrm{B}}$ & \multirow[t]{2}{*}{$1.050^{\mathrm{B}}$} & \multirow[t]{2}{*}{$1.088^{\mathrm{a}}$} \\
\hline & & & & $54755^{\mathrm{b}}$ & & & \\
\hline & \multirow{2}{*}{${ }^{3} \mathrm{~F}_{4}$} & $88422.30^{\mathrm{A}}$ & \multirow[t]{2}{*}{$54664.69^{\mathrm{B}}$} & $55321.35^{a}$ & $1.196^{\mathrm{A}}$ & $1.139^{\mathrm{B}}$ & $1.136^{\mathrm{a}}$ \\
\hline & & $76155.48^{\mathrm{B}}$ & & $55303^{\mathrm{b}}$ & $1.212^{\mathrm{B}}$ & & \\
\hline $5 \mathrm{~d} 6 \mathrm{~d}$ & ${ }^{3} S_{1}$ & - & $54175.01^{\mathrm{B}}$ & $54365.80^{\mathrm{a}}$ & - & $1.566^{\mathrm{B}}$ & $1.455^{\mathrm{a}}$ \\
\hline & & & & $54370^{\mathrm{b}}$ & & & \\
\hline $5 \mathrm{~d} 6 \mathrm{~d}$ & ${ }^{1} S_{0}$ & $74225.09^{\mathrm{B}}$ & $54244.49^{\mathrm{B}}$ & $54793.82^{\mathrm{a}}$ & & & \\
\hline $5 \mathrm{~d} 6 \mathrm{~d}$ & ${ }^{1} \mathrm{D}_{2}$ & $84447.02^{\mathrm{A}}$ & $55024.06^{\mathrm{B}}$ & $55184.05^{\mathrm{a}}$ & $0.996^{\mathrm{A}}$ & $1.056^{\mathrm{B}}$ & $1.183^{\mathrm{a}}$ \\
\hline & & $76453.25^{\mathrm{B}}$ & & $55208^{\mathrm{b}}$ & $1.051^{\mathrm{B}}$ & & \\
\hline $5 \mathrm{~d} 6 \mathrm{~d}$ & ${ }^{3} \mathrm{G}_{3}$ & $69310.22^{\mathrm{A}}$ & $55169.13^{\mathrm{B}}$ & $52857.88^{\mathrm{a}}$ & $0.803^{\mathrm{A}}$ & $0.798^{\mathrm{B}}$ & $0.861^{\mathrm{a}}$ \\
\hline & & $75153.22^{\mathrm{B}}$ & & $52878^{\mathrm{b}}$ & $0.831^{\mathrm{B}}$ & & \\
\hline & ${ }^{3} \mathrm{G}_{4}$ & $70592.95^{\mathrm{A}}$ & $55607.00^{\mathrm{B}}$ & $53333.37^{\mathrm{a}}$ & $1.050^{\mathrm{A}}$ & $1.069^{\mathrm{B}}$ & $1.036^{\mathrm{a}}$ \\
\hline & & $75339.70^{\mathrm{B}}$ & & $53368^{\mathrm{b}}$ & $1.070^{\mathrm{B}}$ & & \\
\hline & ${ }^{3} \mathrm{G}_{5}$ & $71830.19^{\mathrm{A}}$ & $56214.97^{\mathrm{B}}$ & $54434.65^{\mathrm{a}}$ & $1.200^{\mathrm{A}, \mathrm{B}}$ & $1.200^{\mathrm{B}}$ & $1.21^{\mathrm{a}}$ \\
\hline & & $76224.45^{\mathrm{B}}$ & & $54435^{\mathrm{b}}$ & & & \\
\hline $5 \mathrm{~d} 6 \mathrm{~d}$ & ${ }^{3} \mathrm{P}_{0}$ & $90419.13^{\mathrm{A}}$ & $55630.81^{\mathrm{B}}$ & $54964.19^{a}$ & & & \\
\hline & & $76456.13^{\mathrm{B}}$ & & $54786^{\mathrm{b}}$ & & & \\
\hline & ${ }^{3} \mathrm{P}_{1}$ & $90863.55^{\mathrm{A}}$ & $55411.73^{\mathrm{B}}$ & $55230.33^{\mathrm{a}}$ & $1.501^{\mathrm{A}}$ & $1.535^{\mathrm{B}}$ & $1.552^{\mathrm{a}}$ \\
\hline & & $76858.62^{\mathrm{B}}$ & & $55352^{\mathrm{b}}$ & $1.626^{\mathrm{B}}$ & & \\
\hline & ${ }^{3} \mathrm{P}_{2}$ & $91525.97^{\mathrm{A}}$ & $55746.81^{\mathrm{B}}$ & $56036.60^{\mathrm{a}}$ & $1.488^{\mathrm{A}}$ & $1.392^{\mathrm{B}}$ & $1.203^{\mathrm{a}}$ \\
\hline & & $76983.87^{\mathrm{B}}$ & & $56090^{\mathrm{b}}$ & $1.385^{\mathrm{B}}$ & & \\
\hline $5 \mathrm{~d} 6 \mathrm{~d}$ & ${ }^{1} \mathrm{G}_{4}$ & $76564.89^{\mathrm{B}}$ & $59068.02^{\mathrm{B}}$ & $56035.70^{a}$ & $1.018^{\mathrm{B}}$ & $1.056^{\mathrm{B}}$ & $1.027^{\mathrm{a}}$ \\
\hline & & & & $55076^{\mathrm{b}}$ & & & \\
\hline $4 f^{2}$ & ${ }^{3} \mathrm{H}_{4}$ & - & $55201.97^{\mathrm{A}}$ & $55107.25^{a}$ & - & $0.839^{\mathrm{A}}$ & $0.883^{\mathrm{a}}$ \\
\hline & & & $53676.92^{\mathrm{B}}$ & $55079^{\mathrm{b}}$ & & $0.887^{\mathrm{B}}$ & \\
\hline & ${ }^{3} \mathrm{H}_{5}$ & - & $56092.25^{\mathrm{A}}$ & $55982.09^{\mathrm{a}}$ & - & $1.033^{\mathrm{A}, \mathrm{B}}$ & $1.033^{\mathrm{a}}$ \\
\hline & & & $55318.80^{\mathrm{B}}$ & $55995^{\mathrm{b}}$ & & & \\
\hline & ${ }^{3} \mathrm{H}_{6}$ & - & $56863.10^{\mathrm{A}}$ & $56837.94^{\mathrm{a}}$ & - & $1.166^{\mathrm{A}}$ & $1.14^{\mathrm{a}}$ \\
\hline & & & $56547.89^{B}$ & $59845^{\mathrm{b}}$ & & $1.165^{\mathrm{B}}$ & \\
\hline $4 \mathrm{f}^{2}$ & ${ }^{1} \mathrm{G}_{4}$ & - & $56394.91^{\mathrm{A}}$ & $59527.60^{a}$ & - & $0.974^{\mathrm{A}}$ & $1.046^{\mathrm{a}}$ \\
\hline & & & $55328.98^{\mathrm{B}}$ & $59522^{\mathrm{b}}$ & & $1.016^{\mathrm{B}}$ & \\
\hline $4 \mathrm{f}^{2}$ & ${ }^{3} \mathrm{~F}_{2}$ & - & $57396.38^{\mathrm{A}}$ & $57399.58^{\mathrm{a}}$ & - & $0.672^{\mathrm{A}}$ & $0.675^{a}$ \\
\hline & & & $56706.59^{\mathrm{B}}$ & $57385^{\mathrm{b}}$ & & $0.680^{\mathrm{B}}$ & \\
\hline & ${ }^{3} \mathrm{~F}_{3}$ & - & $57884.41^{\mathrm{A}}$ & $57918.50^{\mathrm{a}}$ & - & $1.084^{\mathrm{A}}$ & $1.085^{\mathrm{a}}$ \\
\hline & & & $57513.31^{\mathrm{B}}$ & $57936^{\mathrm{b}}$ & & $1.083^{\mathrm{B}}$ & \\
\hline & ${ }^{3} \mathrm{~F}_{4}$ & - & $58533.37^{\mathrm{A}}$ & $58259.41^{\mathrm{a}}$ & - & $1.237^{\mathrm{A}}$ & $1.196^{\mathrm{a}}$ \\
\hline & & & $58315.69^{\mathrm{B}}$ & $58264^{\mathrm{b}}$ & & $1.184^{\mathrm{B}}$ & \\
\hline $4 f^{2}$ & ${ }^{1} \mathrm{I}_{6}$ & - & $61521.93^{\mathrm{A}}$ & $62408.40^{\mathrm{a}}$ & - & $1.001^{\mathrm{A}}$ & $1.003^{\mathrm{a}}$ \\
\hline & & & $61759.71^{\mathrm{B}}$ & & & $1.002^{\mathrm{B}}$ & \\
\hline $4 f^{2}$ & ${ }^{1} \mathrm{D}_{2}$ & - & $62065.79^{\mathrm{A}}$ & $62026.27^{\mathrm{a}}$ & - & $1.028^{\mathrm{A}}$ & $1.054^{\mathrm{a}}$ \\
\hline & & & $62417.80^{\mathrm{B}}$ & $62029^{\mathrm{b}}$ & & $1.044^{\mathrm{B}}$ & \\
\hline $4 \mathrm{f}^{2}$ & ${ }^{3} \mathrm{P}_{0}$ & - & $63593.22^{\mathrm{A}}$ & $63463.95^{\mathrm{a}}$ & - & & \\
\hline & & & $64001.07^{\mathrm{B}}$ & $63496^{\mathrm{b}}$ & & & \\
\hline & ${ }^{3} \mathrm{P}_{1}$ & - & $63865.10^{\mathrm{A}}$ & $63703.18^{\mathrm{a}}$ & - & $1.501^{\mathrm{A}, \mathrm{B}}$ & $1.471^{\mathrm{a}}$ \\
\hline & & & $64480.52^{\mathrm{B}}$ & $63736^{\mathrm{b}}$ & & & \\
\hline
\end{tabular}


TABLE 2: Continued.

\begin{tabular}{|c|c|c|c|c|c|c|c|}
\hline \multicolumn{2}{|c|}{ Levels } & \multicolumn{3}{|c|}{$E\left(\mathrm{~cm}^{-1}\right)$} & \multicolumn{3}{|c|}{$g$-Factors } \\
\hline \multirow{2}{*}{ Conf. } & \multirow{2}{*}{ Term } & \multicolumn{2}{|c|}{ This work } & \multirow{2}{*}{ Other works } & \multicolumn{2}{|c|}{ This work } & \multirow{2}{*}{ Other works } \\
\hline & & $\mathrm{MCHF}+\mathrm{BP}$ & HFR & & $\mathrm{MCHF}+\mathrm{BP}$ & HFR & \\
\hline & ${ }^{3} \mathrm{P}_{2}$ & - & $64283.97^{\mathrm{A}}$ & $64278.92^{\mathrm{a}}$ & - & $1.467^{\mathrm{A}}$ & $1.414^{\mathrm{a}}$ \\
\hline & & & $65194.71^{\mathrm{B}}$ & $64214^{\mathrm{b}}$ & & $1.449^{\mathrm{B}}$ & \\
\hline \multirow[t]{2}{*}{$4 \mathrm{f}^{2}$} & ${ }^{1} \mathrm{~S}_{0}$ & - & $69782.30^{\mathrm{A}}$ & $69505.06^{\mathrm{a}}$ & - & & \\
\hline & & & $71321.50^{\mathrm{B}}$ & & & & \\
\hline \multirow[t]{3}{*}{$6 p^{2}$} & ${ }^{1} \mathrm{D}_{2}$ & $61118.75^{\mathrm{A}}$ & $59830.40^{\mathrm{B}}$ & $59900.08^{\mathrm{a}}$ & $1.205^{\mathrm{A}}$ & $1.061^{\mathrm{B}}$ & $1.035^{\mathrm{a}}$ \\
\hline & & $63903.98^{\mathrm{B}}$ & & $59899^{\mathrm{b}}$ & $1.013^{\mathrm{B}}$ & & \\
\hline & & $68314.29^{\mathrm{D}}$ & & & $1.005^{\mathrm{D}}$ & & \\
\hline \multirow[t]{9}{*}{$6 p^{2}$} & ${ }^{3} \mathrm{P}_{0}$ & $57741.75^{\mathrm{A}}$ & $60001.21^{\mathrm{B}}$ & $60094.84^{\mathrm{a}}$ & & & \\
\hline & & $57033.69^{\mathrm{B}}$ & & $60091^{\mathrm{b}}$ & & & \\
\hline & & $58691.43^{\mathrm{D}}$ & & & & & \\
\hline & ${ }^{3} \mathrm{P}_{1}$ & $58647.09^{\mathrm{A}}$ & $60514.89^{\mathrm{B}}$ & $61128.83^{\mathrm{a}}$ & $1.501^{\mathrm{A}, \mathrm{B}, \mathrm{D}}$ & $1.502^{\mathrm{B}}$ & $1.528^{\mathrm{a}}$ \\
\hline & & $57752.71^{\mathrm{B}}$ & & $61132^{\mathrm{b}}$ & & & \\
\hline & & $59347.37^{\mathrm{D}}$ & & & & & \\
\hline & ${ }^{3} \mathrm{P}_{2}$ & $59269.90^{A}$ & $61418.50^{\mathrm{B}}$ & $62506.36^{\mathrm{a}}$ & $1.296^{\mathrm{A}}$ & $1.435^{\mathrm{B}}$ & $1.416^{\mathrm{a}}$ \\
\hline & & $58826.49^{\mathrm{B}}$ & & $62504^{\mathrm{b}}$ & $1.488^{\mathrm{B}}$ & & \\
\hline & & $60398.00^{\mathrm{D}}$ & & & $1.496^{\mathrm{D}}$ & & \\
\hline \multirow[t]{3}{*}{$6 p^{2}$} & ${ }^{1} S_{0}$ & $70729.58^{\mathrm{A}}$ & $66977.30^{\mathrm{B}}$ & $66591.91^{a}$ & & & \\
\hline & & $83062.12^{\mathrm{B}}$ & & & & & \\
\hline & & $82414.06^{\mathrm{D}}$ & & & & & \\
\hline \multirow[t]{6}{*}{$6 s 6 d$} & ${ }^{3} \mathrm{D}_{1}$ & $79297.74^{\mathrm{A}}$ & $63251.92^{\mathrm{B}}$ & $64361.28^{\mathrm{a}}$ & $0.499^{\text {A.B }}$ & $0.499^{\mathrm{B}}$ & $0.506^{\mathrm{a}}$ \\
\hline & & $86162.37^{\mathrm{B}}$ & & $64374^{\mathrm{b}}$ & & & \\
\hline & ${ }^{3} \mathrm{D}_{2}$ & $79538.63^{\mathrm{A}}$ & $63833.97^{\mathrm{B}}$ & $64529.90^{\mathrm{a}}$ & $1.133^{\mathrm{A}}$ & $1.165^{\mathrm{B}}$ & $1.217^{\mathrm{a}}$ \\
\hline & & $86172.16^{\mathrm{B}}$ & & $64509^{b}$ & $1.166^{\mathrm{B}}$ & & \\
\hline & ${ }^{3} \mathrm{D}_{3}$ & $80684.92^{\mathrm{A}}$ & $64853.61^{B}$ & $64692.59^{\mathrm{a}}$ & $1.334^{\mathrm{A} . \mathrm{B}}$ & $1.334^{\mathrm{B}}$ & - \\
\hline & & $86191.20^{\mathrm{B}}$ & & $64701^{b}$ & & & \\
\hline $6 s 6 \mathrm{~d}$ & ${ }^{1} \mathrm{D}_{2}$ & $86532.15^{\mathrm{B}}$ & $69906.50^{\mathrm{B}}$ & - & $1.001^{\mathrm{B}}$ & $1.003^{\mathrm{B}}$ & - \\
\hline \multirow[t]{2}{*}{$6 s 7 \mathrm{~s}$} & ${ }^{3} \mathrm{~S}_{1}$ & - & $61127.80^{\mathrm{B}}$ & $60660.18^{\mathrm{a}} ?$ & - & $2.001^{\mathrm{B}}$ & $1.955^{\mathrm{a}}$ \\
\hline & & & & $60660^{\mathrm{b}}$ & & & \\
\hline \multirow[t]{2}{*}{$6 \mathrm{~s} 7 \mathrm{~s}$} & ${ }^{1} \mathrm{~S}_{0}$ & - & $63307.50^{\mathrm{B}}$ & & & & \\
\hline & & & & -parity & & & \\
\hline \multirow[t]{9}{*}{$4 \mathrm{f} 6 \mathrm{~s}$} & ${ }^{3} \mathrm{~F}_{2}^{\mathrm{o}}$ & $14378.61^{\mathrm{A}}$ & $14174.39^{\mathrm{A}}$ & $14147.980^{\mathrm{a}}$ & $0.666^{\mathrm{A}, \mathrm{C}}$ & $0.666^{\mathrm{A}, \mathrm{B}}$ & $0.664^{\mathrm{a}}$ \\
\hline & & $14264.12^{\mathrm{B}}$ & $14267.68^{\mathrm{B}}$ & $14184^{\mathrm{b}}$ & $0.669^{\mathrm{B}}$ & & \\
\hline & & $13925.11^{\mathrm{C}}$ & & & & & \\
\hline & ${ }^{3} \mathrm{~F}_{3}^{\mathrm{o}}$ & $14756.50^{\mathrm{A}}$ & $14283.31^{\mathrm{A}}$ & $14375.170^{\mathrm{a}}$ & $1.060^{\mathrm{A}, \mathrm{C}}$ & $1.055^{\mathrm{A}}$ & $1.056^{\mathrm{a}}$ \\
\hline & & $14858.62^{\mathrm{B}}$ & $14256.22^{\mathrm{B}}$ & $14338^{\mathrm{b}}$ & $1.068^{\mathrm{B}}$ & $1.047^{\mathrm{B}}$ & \\
\hline & & $14291.70^{\mathrm{C}}$ & & & & & \\
\hline & ${ }^{3} \mathrm{~F}_{4}^{\mathrm{o}}$ & $16270.43^{\mathrm{A}}$ & $15651.91^{\mathrm{A}}$ & $15698.740^{\mathrm{a}}$ & $1.250^{\mathrm{A}, \mathrm{B}}$ & $1.250^{\mathrm{A}}$ & $1.247^{\mathrm{a}}$ \\
\hline & & $16250.88^{\mathrm{B}}$ & $15747.51^{\mathrm{B}}$ & $15682^{\mathrm{b}}$ & $1.251^{\mathrm{C}}$ & $1.245^{\mathrm{B}}$ & \\
\hline & & $15785.08^{\mathrm{C}}$ & & & & & \\
\hline \multirow[t]{3}{*}{$4 f 6 s$} & ${ }^{1} \mathrm{~F}_{3}^{\mathrm{o}}$ & $16349.78^{\mathrm{A}}$ & $15822.29^{\mathrm{A}}$ & $15773.770^{\mathrm{a}}$ & $1.023^{\mathrm{A}, \mathrm{C}}$ & $1.028^{\mathrm{A}}$ & $1.017^{\mathrm{a}}$ \\
\hline & & $16448.12^{\mathrm{B}}$ & $15725.69^{\mathrm{B}}$ & $15790^{\mathrm{b}}$ & $1.016^{\mathrm{B}}$ & $1.037^{\mathrm{B}}$ & \\
\hline & & $15889.78^{\mathrm{C}}$ & & & & & \\
\hline \multirow[t]{3}{*}{$4 \mathrm{f} 5 \mathrm{~d}$} & ${ }^{1} \mathrm{G}_{4}^{\mathrm{o}}$ & $23958.81^{\mathrm{A}}$ & $16965.67^{\mathrm{A}}$ & $16559.170^{\mathrm{a}}$ & $0.971^{\mathrm{A}, \mathrm{C}}$ & $0.905^{\mathrm{A}}$ & $0.969^{\mathrm{a}}$ \\
\hline & & $25542.89^{\mathrm{B}}$ & $15525.19^{\mathrm{B}}$ & $16630^{\mathrm{b}}$ & $1.059^{\mathrm{B}}$ & $1.005^{\mathrm{B}}$ & \\
\hline & & $23797.47^{\mathrm{C}}$ & & & & & \\
\hline \multirow[t]{3}{*}{$4 \mathrm{f} 5 \mathrm{~d}$} & ${ }^{3} \mathrm{~F}_{2}^{\mathrm{o}}$ & $25007.46^{\mathrm{A}}$ & $17448.60^{\mathrm{A}}$ & $17211.930^{\mathrm{a}}$ & $0.725^{\mathrm{A}}$ & $0.713^{\mathrm{A}}$ & $0.754^{\mathrm{a}}$ \\
\hline & & $28148.54^{\mathrm{B}}$ & $18658.83^{\mathrm{B}}$ & $17196^{\mathrm{b}}$ & $0.685^{\mathrm{B}}$ & $0.680^{\mathrm{B}}$ & \\
\hline & & $24733.62^{\mathrm{C}}$ & & & $0.735^{\mathrm{C}}$ & & \\
\hline
\end{tabular}


TABLe 2: Continued.

\begin{tabular}{|c|c|c|c|c|c|c|c|}
\hline \multicolumn{2}{|c|}{ Levels } & \multicolumn{3}{|c|}{$E\left(\mathrm{~cm}^{-1}\right)$} & \multicolumn{3}{|c|}{$g$-Factors } \\
\hline \multirow{2}{*}{ Conf. } & \multirow{2}{*}{ Term } & \multicolumn{2}{|c|}{ This work } & \multirow{2}{*}{ Other works } & \multicolumn{2}{|c|}{ This work } & \multirow{2}{*}{ Other works } \\
\hline & & $\mathrm{MCHF}+\mathrm{BP}$ & HFR & & $\mathrm{MCHF}+\mathrm{BP}$ & HFR & \\
\hline & ${ }^{3} \mathrm{~F}_{3}^{\mathrm{o}}$ & $25740.72^{\mathrm{A}}$ & $18242.30^{\mathrm{A}}$ & $18235.560^{\mathrm{a}}$ & $1.091^{\mathrm{A}}$ & $1.083^{\mathrm{A}}$ & $1.086^{\mathrm{a}}$ \\
\hline & & $29158.71^{\mathrm{B}}$ & $18866.08^{\text {B }}$ & $18215^{\mathrm{b}}$ & $1.083^{\mathrm{B}}$ & $1.079^{\mathrm{B}}$ & \\
\hline & & $25479.83^{C}$ & & & $1.098^{\mathrm{C}}$ & & \\
\hline & ${ }^{3} \mathrm{~F}_{4}^{\mathrm{o}}$ & $27201.89^{\mathrm{A}}$ & $19267.37^{\mathrm{A}}$ & $19214.540^{\mathrm{a}}$ & $1.225^{\mathrm{A}}$ & $1.226^{\mathrm{A}}$ & $1.232^{\mathrm{a}}$ \\
\hline & & $30358.80^{\mathrm{B}}$ & $19233.10^{\text {B }}$ & $19199^{\mathrm{b}}$ & $1.241^{\mathrm{B}}$ & $1.241^{\mathrm{B}}$ & \\
\hline & & $26932.00^{\mathrm{C}}$ & & & $1.224^{\mathrm{C}}$ & & \\
\hline \multirow[t]{8}{*}{$4 \mathrm{f} 5 \mathrm{~d}$} & ${ }^{3} \mathrm{H}_{4}^{\mathrm{o}}$ & $25669.19^{\mathrm{A}}$ & $17727.74^{\mathrm{A}}$ & $17825.620^{\mathrm{a}}$ & $0.855^{\mathrm{A}}$ & $0.920^{\mathrm{A}}$ & $0.846^{\mathrm{a}}$ \\
\hline & & $27280.97^{\mathrm{B}}$ & $17683.40^{\mathrm{B}}$ & $17803^{\mathrm{b}}$ & $0.862^{\mathrm{B}}$ & $0.806^{\mathrm{B}}$ & \\
\hline & & $25499.67^{\mathrm{C}}$ & & & $0.856^{\mathrm{C}}$ & & \\
\hline & ${ }^{3} \mathrm{H}_{5}^{\circ}$ & $26756.81^{\mathrm{A}}$ & $18250.23^{\mathrm{A}}$ & $18580.410^{\mathrm{a}}$ & $1.033^{\mathrm{A}, \mathrm{B}, \mathrm{C}}$ & $1.034^{\mathrm{A}, \mathrm{B}}$ & $1.017^{\mathrm{a}}$ \\
\hline & & $28223.06^{\mathrm{B}}$ & $18122.03^{\mathrm{B}}$ & $18573^{\mathrm{b}}$ & & & \\
\hline & & $26582.89^{C}$ & & & & & \\
\hline & ${ }^{3} \mathrm{H}_{6}^{\mathrm{o}}$ & $28460.49^{\mathrm{A}}$ & $19394.18^{\mathrm{A}}$ & $19749.620^{\mathrm{a}}$ & $1.167^{\mathrm{A}, \mathrm{C}}$ & $1.167^{\mathrm{A}, \mathrm{B}}$ & $1.178^{\mathrm{a}}$ \\
\hline & & $28275.22^{\mathrm{C}}$ & $18802.98^{\mathrm{B}}$ & $19767^{\mathrm{b}}$ & & & \\
\hline \multirow[t]{3}{*}{$4 \mathrm{f} 5 \mathrm{~d}$} & ${ }^{1} \mathrm{D}_{2}^{\mathrm{o}}$ & $31071.25^{\mathrm{A}}$ & $19151.58^{\mathrm{A}}$ & $18895.410^{\mathrm{a}}$ & $0.981^{\mathrm{A}}$ & $0.970^{\mathrm{A}}$ & $0.923^{\mathrm{a}}$ \\
\hline & & $32636.97^{\mathrm{B}}$ & $21057.91^{\mathrm{B}}$ & $18926^{\mathrm{b}}$ & $0.997^{\mathrm{B}}$ & $1.114^{\mathrm{B}}$ & \\
\hline & & $30916.96^{\mathrm{C}}$ & & & $0.982^{\mathrm{C}}$ & & \\
\hline \multirow[t]{9}{*}{$4 f 5 d$} & ${ }^{3} \mathrm{G}_{3}^{\mathrm{o}}$ & $31665.41^{\mathrm{A}}$ & $20315.59^{A}$ & $20402.820^{\mathrm{a}}$ & $0.763^{\mathrm{A}}$ & $0.757^{\mathrm{A}}$ & $0.757^{\mathrm{a}}$ \\
\hline & & $34021.44^{\mathrm{B}}$ & $21064.80^{\mathrm{B}}$ & $20405^{\mathrm{b}}$ & $0.758^{\mathrm{B}}$ & $0.782^{\mathrm{B}}$ & \\
\hline & & $31554.09^{\mathrm{C}}$ & & & $0.764^{\mathrm{C}}$ & & \\
\hline & ${ }^{3} \mathrm{G}_{4}^{\mathrm{o}}$ & $32976.84^{\mathrm{A}}$ & $21247.80^{\mathrm{A}}$ & $21331.600^{\mathrm{a}}$ & $1.050^{A, B, C}$ & $1.049^{\mathrm{A}}$ & $1.049^{\mathrm{a}}$ \\
\hline & & $35195.62^{\mathrm{B}}$ & $21629.90^{B}$ & $21324^{\mathrm{b}}$ & & $1.054^{\mathrm{B}}$ & \\
\hline & & $32859.39^{\mathrm{C}}$ & & & & & \\
\hline & ${ }^{3} \mathrm{G}_{5}^{\mathrm{o}}$ & $34349.12^{\mathrm{A}}$ & $22107.93^{A}$ & $22282.900^{\mathrm{a}}$ & $1.200^{\mathrm{A}, \mathrm{B}, \mathrm{C}}$ & $1.200^{\mathrm{A}}$ & $1.197^{\mathrm{a}}$ \\
\hline & & $36498.47^{\mathrm{B}}$ & $21982.01^{B}$ & $22269^{\mathrm{b}}$ & & $1.199^{\mathrm{B}}$ & \\
\hline & & $34188.45^{\mathrm{C}}$ & & & & & \\
\hline \multirow[t]{9}{*}{$4 f 5 d$} & ${ }^{3} \mathrm{D}_{1}^{\mathrm{o}}$ & $35406.25^{\mathrm{A}}$ & $21395.42^{\mathrm{A}}$ & $21441.730^{\mathrm{a}}$ & $0.503^{\mathrm{A}}$ & $0.508^{\mathrm{A}}$ & $0.542^{\mathrm{a}}$ \\
\hline & & $37453.79^{\mathrm{B}}$ & $22982.69^{B}$ & $21477^{\mathrm{b}}$ & $0.501^{B}$ & $0.591^{\mathrm{B}}$ & \\
\hline & & $35168.59^{\mathrm{C}}$ & & & $0.512^{\mathrm{C}}$ & & \\
\hline & ${ }^{3} \mathrm{D}_{2}^{\circ}$ & $35923.09^{\mathrm{A}}$ & $22032.81^{\mathrm{A}}$ & $22106.020^{\mathrm{a}}$ & $1.170^{\mathrm{A}}$ & $1.159^{\mathrm{A}}$ & $1.167^{\mathrm{a}}$ \\
\hline & & $37936.33^{\mathrm{B}}$ & $23003.68^{\mathrm{B}}$ & $22112^{\mathrm{b}}$ & $1.169^{\mathrm{B}, \mathrm{C}}$ & $1.195^{\mathrm{B}}$ & \\
\hline & & $35808.76^{C}$ & & & & & \\
\hline & ${ }^{3} \mathrm{D}_{3}^{\mathrm{o}}$ & $36203.19^{A}$ & $22475.18^{A}$ & $22537.300^{\mathrm{a}}$ & $1.264^{\mathrm{A}}$ & $1.305^{\mathrm{A}}$ & $1.288^{\mathrm{a}}$ \\
\hline & & $38173.89^{\mathrm{B}}$ & $23296.42^{\mathrm{B}}$ & $22483^{\mathrm{b}}$ & $1.270^{\mathrm{B}}$ & $1.251^{\mathrm{B}}$ & \\
\hline & & $36085.58^{\mathrm{C}}$ & & & $1.266^{\mathrm{C}}$ & & \\
\hline \multirow[t]{9}{*}{$4 \mathrm{f} 5 \mathrm{~d}$} & ${ }^{3} \mathrm{P}_{0}^{\mathrm{o}}$ & $39198.12^{\mathrm{A}}$ & $22445.78^{A}$ & $22683.700^{\mathrm{a}}$ & & & \\
\hline & & $40330.43^{\mathrm{B}}$ & $22463.20^{\mathrm{B}}$ & $22674^{\mathrm{b}}$ & & & \\
\hline & & $38565.29^{C}$ & & & & & \\
\hline & ${ }^{3} \mathrm{P}_{1}^{\mathrm{o}}$ & $39440.20^{\mathrm{A}}$ & $22667.40^{\mathrm{A}}$ & $22705.150^{\mathrm{a}}$ & $1.500^{\mathrm{A}}$ & $1.481^{\mathrm{A}}$ & $1.431^{\mathrm{a}}$ \\
\hline & & $40455.15^{\mathrm{B}}$ & $22524.24^{\mathrm{B}}$ & $22690^{\mathrm{b}}$ & $1.498^{\mathrm{B}}$ & $1.345^{\mathrm{B}}$ & \\
\hline & & $38560.21^{\mathrm{C}}$ & & & $1.485^{\mathrm{C}}$ & & \\
\hline & ${ }^{3} \mathrm{P}_{2}^{\mathrm{o}}$ & $40081.21^{\mathrm{A}}$ & $23571.92^{\mathrm{A}}$ & $23246.930^{\mathrm{a}}$ & $1.497^{\mathrm{A}}$ & $1.489^{\mathrm{A}}$ & $1.459^{\mathrm{a}}$ \\
\hline & & $40802.14^{\mathrm{B}}$ & $21850.66^{\mathrm{B}}$ & $23294^{\mathrm{b}}$ & $1.492^{\mathrm{B}, \mathrm{C}}$ & $1.337^{\mathrm{B}}$ & \\
\hline & & $39052.77^{\mathrm{C}}$ & & & & & \\
\hline \multirow[t]{3}{*}{$4 \mathrm{f} 5 \mathrm{~d}$} & ${ }^{1} \mathrm{~F}_{3}^{\mathrm{o}}$ & $38465.61^{\mathrm{A}}$ & $24240.21^{\mathrm{A}}$ & $24552.700^{\mathrm{a}}$ & $1.061^{\mathrm{A}}$ & $1.024^{\mathrm{A}}$ & $1.034^{\mathrm{a}}$ \\
\hline & & $40442.67^{\mathrm{B}}$ & $22482.21^{\mathrm{B}}$ & $24569^{\mathrm{b}}$ & $1.057^{\mathrm{B}}$ & $1.055^{\mathrm{B}}$ & \\
\hline & & $38356.86^{\mathrm{C}}$ & & & $1.059^{\mathrm{C}}$ & & \\
\hline
\end{tabular}


TABLe 2: Continued.

\begin{tabular}{|c|c|c|c|c|c|c|c|}
\hline \multicolumn{2}{|c|}{ Levels } & \multicolumn{3}{|c|}{$E\left(\mathrm{~cm}^{-1}\right)$} & \multicolumn{3}{|c|}{$g$-Factors } \\
\hline \multirow{2}{*}{ Conf. } & \multirow{2}{*}{ Term } & \multicolumn{2}{|c|}{ This work } & \multirow{2}{*}{ Other works } & \multicolumn{2}{|c|}{ This work } & \multirow{2}{*}{ Other works } \\
\hline & & $\mathrm{MCHF}+\mathrm{BP}$ & HFR & & $\mathrm{MCHF}+\mathrm{BP}$ & HFR & \\
\hline \multirow[t]{2}{*}{$4 \mathrm{ff} 5 \mathrm{~d}$} & ${ }^{1} \mathrm{H}_{5}^{\mathrm{o}}$ & $46448.76^{\mathrm{C}}$ & $28811.47^{\mathrm{A}}$ & $28525.710^{\mathrm{a}}$ & $1.000^{\mathrm{C}}$ & $1.001^{\mathrm{A}, \mathrm{B}}$ & $1.004^{\mathrm{a}}$ \\
\hline & & & $27193.29^{\mathrm{B}}$ & $28529^{\mathrm{b}}$ & & & \\
\hline \multirow[t]{2}{*}{$4 \mathrm{f} 5 \mathrm{~d}$} & ${ }^{1} \mathrm{P}_{1}^{\mathrm{o}}$ & $47396.25^{\mathrm{C}}$ & $30265.31^{\mathrm{A}}$ & $30353.330^{\mathrm{a}}$ & $1.004^{\mathrm{C}}$ & $1.040^{\mathrm{A}}$ & $1.074^{\mathrm{a}}$ \\
\hline & & & $21477.10^{\mathrm{B}}$ & & & $1.060^{\mathrm{B}}$ & \\
\hline \multirow[t]{3}{*}{$5 d 6 p$} & ${ }^{1} \mathrm{D}_{2}^{\mathrm{o}}$ & $22996.58^{\mathrm{A}}$ & $24514.82^{\mathrm{A}}$ & $24462.66^{\mathrm{a}}$ & $0.969^{\mathrm{A}}$ & $1.014^{\mathrm{A}}$ & $0.887^{\mathrm{a}}$ \\
\hline & & $19478.98^{\mathrm{B}}$ & $24310.90^{\mathrm{B}}$ & $24567^{\mathrm{b}}$ & $1.011^{\mathrm{B}}$ & $1.017^{\mathrm{B}}$ & \\
\hline & & $22618.34^{\mathrm{C}}$ & & & $0.975^{\mathrm{C}}$ & & \\
\hline \multirow[t]{9}{*}{$5 \mathrm{~d} 6 \mathrm{p}$} & ${ }^{3} \mathrm{D}_{1}^{\mathrm{o}}$ & $24485.41^{\mathrm{A}}$ & $25907.37^{\mathrm{A}}$ & $25973.37^{\mathrm{a}}$ & $0.502^{\mathrm{A}}$ & $0.579^{\mathrm{A}}$ & $0.782^{\mathrm{a}}$ \\
\hline & & $20646.65^{\mathrm{B}}$ & $25815.48^{\mathrm{B}}$ & $25839^{\mathrm{b}}$ & $0.500^{\mathrm{B}}$ & $0.507^{\mathrm{B}}$ & \\
\hline & & $23978.78^{C}$ & & & $0.512^{\mathrm{C}}$ & & \\
\hline & ${ }^{3} \mathrm{D}_{2}^{\mathrm{o}}$ & $25339.58^{A}$ & $26457.83^{\mathrm{A}}$ & $27388.11^{\mathrm{a}}$ & $1.161^{\mathrm{A}, \mathrm{B}}$ & $1.133^{\mathrm{A}}$ & $1.168^{\mathrm{a}}$ \\
\hline & & $21467.30^{\mathrm{B}}$ & $26411.29^{\mathrm{B}}$ & $27362^{\mathrm{b}}$ & $1.139^{\mathrm{C}}$ & $1.120^{\mathrm{B}}$ & \\
\hline & & $24934.60^{\mathrm{C}}$ & & & & & \\
\hline & ${ }^{3} \mathrm{D}_{3}^{\mathrm{o}}$ & $26382.52^{\mathrm{A}}$ & $26950.18^{A}$ & $28315.25^{\mathrm{a}}$ & $1.323^{\mathrm{A}}$ & $1.316^{\mathrm{A}}$ & $1.308^{\mathrm{a}}$ \\
\hline & & $22400.30^{\mathrm{B}}$ & $26951.83^{\text {B }}$ & $28290^{\mathrm{b}}$ & $1.332^{\mathrm{B}}$ & $1.312^{\mathrm{B}}$ & \\
\hline & & $25902.53^{C}$ & & & $1.316^{\mathrm{C}}$ & & \\
\hline \multirow[t]{2}{*}{$5 \mathrm{~d} 6 \mathrm{p}$} & ${ }^{1} \mathrm{P}_{1}^{\mathrm{o}}$ & - & $26059.89^{A}$ & - & - & $0.955^{\mathrm{A}}$ & - \\
\hline & & & $30735.20^{\mathrm{B}}$ & & & $1.064^{\mathrm{B}}$ & \\
\hline \multirow[t]{9}{*}{$5 d 6 p$} & ${ }^{3} \mathrm{~F}_{2}^{\mathrm{o}}$ & $27730.01^{\mathrm{A}}$ & $27524.69^{\mathrm{A}}$ & $26414.01^{\mathrm{a}}$ & $0.702^{\mathrm{A}}$ & $0.691^{\mathrm{A}}$ & $0.825^{\mathrm{a}}$ \\
\hline & & $23795.02^{\mathrm{B}}$ & $27633.61^{\mathrm{B}}$ & $26409^{\mathrm{b}}$ & $0.700^{\mathrm{B}}$ & $0.705^{\mathrm{B}}$ & \\
\hline & & $27441.41^{\mathrm{C}}$ & & & $0.693^{\mathrm{C}}$ & & \\
\hline & ${ }^{3} \mathrm{~F}_{3}^{\mathrm{o}}$ & $28937.43^{\mathrm{A}}$ & $27941.30^{\mathrm{A}}$ & $26837.66^{\mathrm{a}}$ & $1.083^{\mathrm{A}, \mathrm{B}, \mathrm{C}}$ & $1.098^{\mathrm{A}}$ & $1.088^{\mathrm{a}}$ \\
\hline & & $24648.21^{\mathrm{B}}$ & $28199.61^{\mathrm{B}}$ & $26828^{\mathrm{b}}$ & & $1.103^{\mathrm{B}}$ & \\
\hline & & $28616.27^{\mathrm{C}}$ & & & & & \\
\hline & ${ }^{3} \mathrm{~F}_{4}^{\mathrm{o}}$ & $30401.18^{\mathrm{A}}$ & $28617.50^{\mathrm{A}}$ & $28565.40^{\mathrm{a}}$ & $1.248^{\mathrm{A}, \mathrm{C}}$ & $1.251^{\mathrm{A}, \mathrm{B}}$ & $1.245^{\mathrm{a}}$ \\
\hline & & $26075.37^{\mathrm{B}}$ & $29206.78^{B}$ & $28531^{\mathrm{b}}$ & $1.138^{\mathrm{B}}$ & & \\
\hline & & $30028.17^{\mathrm{C}}$ & & & & & \\
\hline \multirow[t]{9}{*}{$5 \mathrm{~d} 6 \mathrm{p}$} & ${ }^{3} \mathrm{P}_{0}^{\mathrm{o}}$ & $27489.54^{\mathrm{A}}$ & $31804.81^{\mathrm{A}}$ & $31785.82^{\mathrm{a}}$ & & & \\
\hline & & $23324.32^{\mathrm{B}}$ & $32009.00^{\mathrm{B}}$ & $31797^{\mathrm{b}}$ & & & \\
\hline & & $27777.75^{\mathrm{C}}$ & & & & & \\
\hline & ${ }^{3} \mathrm{P}_{1}^{\mathrm{o}}$ & $27767.31^{A}$ & $32243.52^{\mathrm{A}}$ & $32160.99^{\mathrm{a}}$ & $1.498^{\mathrm{A}}$ & $1.497^{\mathrm{A}}$ & $1.492^{\mathrm{a}}$ \\
\hline & & $23622.98^{\text {B }}$ & $32711.11^{\mathrm{B}}$ & $32134^{\mathrm{b}}$ & $1.500^{\mathrm{B}}$ & $1.437^{\mathrm{B}}$ & \\
\hline & & $27808.39^{\mathrm{C}}$ & & & $1.473^{\mathrm{C}}$ & & \\
\hline & ${ }^{3} \mathrm{P}_{2}^{\mathrm{o}}$ & $28480.17^{\mathrm{A}}$ & $33269.26^{\mathrm{A}}$ & $29593^{\mathrm{b}}$ & $1.469^{\mathrm{A}}$ & $1.501^{\mathrm{A}}$ & - \\
\hline & & $24351.70^{\mathrm{B}}$ & $33441.59^{\mathrm{B}}$ & & $1.460^{\mathrm{B}}$ & $1.500^{\mathrm{B}}$ & \\
\hline & & $28624.94^{\mathrm{C}}$ & & & $1.482^{\mathrm{C}}$ & & \\
\hline \multirow[t]{3}{*}{$5 d 6 p$} & ${ }^{1} \mathrm{~F}_{3}^{\mathrm{o}}$ & $34048.66^{\mathrm{A}}$ & $32144.41^{\mathrm{A}}$ & $32201.05^{\mathrm{a}}$ & $0.999^{\mathrm{A}}$ & $1.002^{\mathrm{A}}$ & $1.005^{\mathrm{a}}$ \\
\hline & & $31107.39^{\mathrm{B}}$ & $32749.00^{\mathrm{B}}$ & $32273^{\mathrm{b}}$ & $1.000^{\mathrm{B}}$ & $1.003^{\mathrm{B}}$ & \\
\hline & & $33748.76^{\mathrm{C}}$ & & & $0.997^{\mathrm{C}}$ & & \\
\hline \multirow[t]{6}{*}{$6 s 6 p$} & ${ }^{3} \mathrm{P}_{0}^{\mathrm{o}}$ & $36704.74^{\mathrm{A}}$ & $27560.27^{\mathrm{A}}$ & $27545.850^{\mathrm{a}}$ & & & \\
\hline & & $32174.15^{\mathrm{B}}$ & $28370.01^{\mathrm{B}}$ & $27563^{\mathrm{b}}$ & & & \\
\hline & ${ }^{3} \mathrm{P}_{1}^{\mathrm{o}}$ & $37150.85^{\mathrm{A}}$ & $28103.04^{\mathrm{A}}$ & $28154.550^{\mathrm{a}} ?$ & $1.498^{\mathrm{A}}$ & $1.438^{\mathrm{A}}$ & $1.267^{\mathrm{a}}$ \\
\hline & & $32612.03^{\mathrm{B}}$ & $28403.35^{\mathrm{B}}$ & $28147^{\mathrm{b}}$ & $1.501^{\mathrm{B}}$ & $1.496^{\mathrm{B}}$ & \\
\hline & ${ }^{3} \mathrm{P}_{2}^{\mathrm{o}}$ & $37997.38^{\mathrm{A}}$ & $29477.41^{\mathrm{A}}$ & $29498.050^{\mathrm{a}} ?$ & $1.494^{\mathrm{A}}$ & $1.500^{\mathrm{A}}$ & $1.471^{\mathrm{a}}$ \\
\hline & & $33501.99^{\mathrm{B}}$ & $28508.94^{\mathrm{B}}$ & $33133^{\mathrm{b}}$ & $1.491^{\mathrm{B}}$ & $1.501^{\mathrm{B}}$ & \\
\hline $6 s 6 p$ & ${ }^{1} \mathrm{P}_{1}^{\mathrm{o}}$ & - & $45700.40^{\mathrm{A}}$ & $45692.170^{\mathrm{a}}$ & - & $1.002^{\mathrm{A}}$ & $0.999^{\mathrm{a}}$ \\
\hline
\end{tabular}


TABLE 2: Continued.

\begin{tabular}{|c|c|c|c|c|c|c|c|}
\hline \multicolumn{2}{|c|}{ Levels } & \multicolumn{3}{|c|}{$E\left(\mathrm{~cm}^{-1}\right)$} & \multicolumn{3}{|c|}{$g$-Factors } \\
\hline \multirow{2}{*}{ Conf. } & \multirow{2}{*}{ Term } & \multicolumn{2}{|c|}{ This work } & \multirow{2}{*}{ Other works } & \multicolumn{2}{|c|}{ This work } & \multirow{2}{*}{ Other works } \\
\hline & & $\mathrm{MCHF}+\mathrm{BP}$ & HFR & & $\mathrm{MCHF}+\mathrm{BP}$ & HFR & \\
\hline & & & $45953.40^{\mathrm{B}}$ & $45702^{b}$ & & $1.121^{\mathrm{B}}$ & \\
\hline \multirow[t]{3}{*}{$4 \mathrm{f} 7 \mathrm{~s}$} & ${ }^{3} \mathrm{~F}_{2}^{\mathrm{o}}$ & - & $60463.77^{\mathrm{B}}$ & - & - & $0.667^{\mathrm{B}}$ & - \\
\hline & ${ }^{3} \mathrm{~F}_{3}^{\mathrm{o}}$ & - & $60470.13^{\mathrm{B}}$ & - & - & $1.048^{\mathrm{B}}$ & - \\
\hline & ${ }^{3} \mathrm{~F}_{4}^{\mathrm{o}}$ & - & $61873.10^{\mathrm{B}}$ & - & - & $1.250^{\mathrm{B}}$ & - \\
\hline $4 \mathrm{f} 7 \mathrm{~s}$ & ${ }^{1} \mathrm{~F}_{3}^{\mathrm{o}}$ & - & $61880.70^{\mathrm{B}}$ & - & - & $1.036^{\mathrm{B}}$ & - \\
\hline \multirow[t]{3}{*}{$4 \mathrm{f} 6 \mathrm{~d}$} & ${ }^{3} \mathrm{~F}_{2}^{\mathrm{o}}$ & - & $57457.37^{\mathrm{B}}$ & - & - & $0.798^{\mathrm{B}}$ & - \\
\hline & ${ }^{3} \mathrm{~F}_{3}^{\mathrm{o}}$ & - & $59105.69^{B}$ & - & - & $1.121^{\mathrm{B}}$ & - \\
\hline & ${ }^{3} \mathrm{~F}_{4}^{\mathrm{o}}$ & - & $61261.54^{\mathrm{B}}$ & - & - & $1.135^{\mathrm{B}}$ & - \\
\hline $4 \mathrm{f} 6 \mathrm{~d}$ & ${ }^{1} \mathrm{G}_{4}^{\mathrm{o}}$ & - & $59353.91^{\mathrm{B}}$ & - & - & $1.037^{\mathrm{B}}$ & - \\
\hline \multirow[t]{3}{*}{$4 \mathrm{f} 6 \mathrm{~d}$} & ${ }^{3} \mathrm{G}_{3}^{\mathrm{o}}$ & - & $57698.08^{\mathrm{B}}$ & - & - & $0.854^{\mathrm{B}}$ & - \\
\hline & ${ }^{3} \mathrm{G}_{4}^{\mathrm{o}}$ & - & $60092.42^{\mathrm{B}}$ & - & - & $0.998^{\mathrm{B}}$ & - \\
\hline & ${ }^{3} \mathrm{G}_{5}^{\mathrm{o}}$ & - & $61115.66^{\mathrm{B}}$ & - & - & $1.082^{\mathrm{B}}$ & - \\
\hline \multirow[t]{3}{*}{$4 \mathrm{f} 6 \mathrm{~d}$} & ${ }^{3} \mathrm{D}_{1}^{\mathrm{o}}$ & - & $58144.14^{\mathrm{B}}$ & - & - & $0.823^{\mathrm{B}}$ & - \\
\hline & ${ }^{3} \mathrm{D}_{2}^{\mathrm{o}}$ & - & $59943.80^{\mathrm{B}}$ & - & - & $1.028^{\mathrm{B}}$ & - \\
\hline & ${ }^{3} \mathrm{D}_{3}^{\mathrm{o}}$ & - & $61591.58^{\mathrm{B}}$ & - & - & $1.177^{\mathrm{B}}$ & - \\
\hline \multirow[t]{3}{*}{$4 \mathrm{f} 6 \mathrm{~d}$} & ${ }^{3} \mathrm{H}_{4}^{\mathrm{o}}$ & - & $58359.62^{\mathrm{B}}$ & - & - & $0.930^{\mathrm{B}}$ & - \\
\hline & ${ }^{3} \mathrm{H}_{5}^{\mathrm{o}}$ & - & $60449.51^{\mathrm{B}}$ & - & - & $1.081^{\mathrm{B}}$ & - \\
\hline & ${ }^{3} \mathrm{H}_{6}^{\mathrm{o}}$ & - & $62719.38^{\mathrm{B}}$ & - & - & $1.167^{\mathrm{B}}$ & - \\
\hline \multirow[t]{3}{*}{$4 \mathrm{f} 6 \mathrm{~d}$} & ${ }^{3} \mathrm{P}_{0}^{\mathrm{o}}$ & - & $60580.29^{B}$ & - & - & & \\
\hline & ${ }^{3} \mathrm{P}_{1}^{\mathrm{o}}$ & - & $60380.02^{\mathrm{B}}$ & - & - & $1.088^{\mathrm{B}}$ & - \\
\hline & ${ }^{3} \mathrm{P}_{2}^{\mathrm{o}}$ & - & $59703.78^{\mathrm{B}}$ & - & - & $1.279^{\mathrm{B}}$ & - \\
\hline $4 \mathrm{f} 6 \mathrm{~d}$ & ${ }^{1} \mathrm{~F}_{3}^{\mathrm{o}}$ & - & $59802.99^{\mathrm{B}}$ & - & - & $1.015^{\mathrm{B}}$ & - \\
\hline $4 \mathrm{f} 6 \mathrm{~d}$ & ${ }^{1} \mathrm{D}_{2}^{o}$ & - & $61840.89^{B}$ & - & - & $1.233^{\mathrm{B}}$ & - \\
\hline $4 \mathrm{f} 6 \mathrm{~d}$ & ${ }^{1} \mathrm{P}_{1}^{\mathrm{o}}$ & - & $62234.11^{\mathrm{B}}$ & - & - & $1.106^{\mathrm{B}}$ & - \\
\hline $4 \mathrm{f} 6 \mathrm{~d}$ & ${ }^{1} \mathrm{H}_{5}^{\mathrm{o}}$ & - & $62355.62^{B}$ & - & - & $1.071^{\mathrm{B}}$ & - \\
\hline \multirow[t]{3}{*}{$5 \mathrm{~d} 7 \mathrm{p}$} & ${ }^{3} \mathrm{D}_{1}^{\mathrm{o}}$ & - & $44866.71^{\mathrm{B}}$ & - & - & $0.749^{\mathrm{B}}$ & - \\
\hline & ${ }^{3} \mathrm{D}_{2}^{\mathrm{o}}$ & - & $44866.75^{\mathrm{B}}$ & - & - & $1.141^{\mathrm{B}}$ & - \\
\hline & ${ }^{3} \mathrm{D}_{3}^{\mathrm{o}}$ & - & $47740.96^{\mathrm{B}}$ & - & - & $1.267^{\mathrm{B}}$ & - \\
\hline \multirow[t]{3}{*}{$5 \mathrm{~d} 7 \mathrm{p}$} & ${ }^{3} \mathrm{~F}_{2}^{\mathrm{o}}$ & - & $44842.48^{\mathrm{B}}$ & - & - & $0.691^{\mathrm{B}}$ & - \\
\hline & ${ }^{3} \mathrm{~F}_{3}^{\mathrm{o}}$ & - & $44879.84^{\mathrm{B}}$ & - & - & $1.068^{\mathrm{B}}$ & - \\
\hline & ${ }^{3} \mathrm{~F}_{4}^{\mathrm{o}}$ & - & $47724.49^{\mathrm{B}}$ & - & - & $1.251^{\mathrm{B}}$ & - \\
\hline \multirow[t]{3}{*}{$5 \mathrm{~d} 7 \mathrm{p}$} & ${ }^{3} \mathrm{P}_{0}^{\mathrm{o}}$ & - & $44877.71^{\mathrm{B}}$ & - & - & & \\
\hline & ${ }^{3} \mathrm{P}_{1}^{\mathrm{o}}$ & - & $44424.70^{\mathrm{B}}$ & - & - & $1.073^{\mathrm{B}}$ & - \\
\hline & ${ }^{3} \mathrm{P}_{2}^{\mathrm{o}}$ & - & $47745.11^{\mathrm{B}}$ & - & - & $1.271^{\mathrm{B}}$ & - \\
\hline $5 \mathrm{~d} 7 \mathrm{p}$ & ${ }^{1} \mathrm{D}_{2}^{\mathrm{o}}$ & - & $47754.57^{\mathrm{B}}$ & - & - & $1.230^{\mathrm{B}}$ & - \\
\hline $5 \mathrm{~d} 7 \mathrm{p}$ & ${ }^{1} \mathrm{~F}_{3}^{\mathrm{o}}$ & - & $47767.78^{\mathrm{B}}$ & - & - & $1.083^{\mathrm{B}}$ & - \\
\hline $5 \mathrm{~d} 7 \mathrm{p}$ & ${ }^{1} \mathrm{P}_{1}^{\mathrm{o}}$ & - & $48313.70^{\mathrm{B}}$ & - & - & $1.057^{\mathrm{B}}$ & - \\
\hline \multirow[t]{3}{*}{$6 s 7 \mathrm{p}$} & ${ }^{3} \mathrm{P}_{0}^{\mathrm{o}}$ & - & $59907.82^{\mathrm{B}}$ & - & - & & \\
\hline & ${ }^{3} \mathrm{P}_{1}^{\mathrm{o}}$ & - & $60242.28^{\mathrm{B}}$ & - & - & $1.451^{\mathrm{B}}$ & - \\
\hline & ${ }^{3} \mathrm{P}_{2}^{\mathrm{o}}$ & - & $61529.70^{\mathrm{B}}$ & - & - & $1.495^{\mathrm{B}}$ & - \\
\hline $6 s 7 p$ & ${ }^{1} \mathrm{P}_{1}^{\mathrm{o}}$ & - & $63316.70^{\mathrm{B}}$ & - & - & $1.033^{\mathrm{B}}$ & - \\
\hline
\end{tabular}

${ }^{a}$ Reference [28], ${ }^{\mathrm{b}}$ reference [12], ? reference [28].

good for $5 \mathrm{~d} 6 \mathrm{~s}^{3} \mathrm{D}$ levels obtained from calculation $\mathrm{C}$ and $5 \mathrm{~d} 6 \mathrm{~s}$ ${ }^{1} \mathrm{D}$ level obtained from calculation A. The result obtained from calculation $\mathrm{D}$ for $6 \mathrm{~s}^{2}$ level and the results obtained from calculation $\mathrm{A}$ for $6 \mathrm{p}^{2}$ are in agreement when comparing with others. The results obtained from calculations $\mathrm{A}$ and $\mathrm{B}$ are also somewhat in agreement for $5 \mathrm{~d} 6 \mathrm{~d}$ and $6 \mathrm{~s} 6 \mathrm{~d}$ levels. $4 \mathrm{~s} 6 \mathrm{~s}$, $5 \mathrm{~d} 6 \mathrm{p}$, and $5 \mathrm{~d} 6 \mathrm{p}$ levels are good in agreement with others. The $4 \mathrm{f} 5 \mathrm{~d}$ level is somewhat poor according to other works. It can be said that these cases occur due to unfilled $d$ and, in particular, $f$ subshells. The configuration including these 
subshells complicates the calculations in the MCHF method. Results that obtained the Landé $g$-factors are in quite good agreement with others.

We have studied with two configuration sets (in Table 1) for considering correlation effects in the HFR calculations performed using Cowan's computer code [45]. These configuration sets are also given in Table 1. This approach, although based on the Schrödinger equation, includes the relativistic effects like the mass-velocity corrections and the Darwin contribution beside the spin-orbit effect. In these calculations, the HFR method was combined with a least-squares optimization routine minimizing the discrepancies between observed and calculated energy levels. The scaling factors of the Slater parameters $\left(F^{k}\right.$ and $\left.G^{k}\right)$ and of configuration interaction integrals $\left(R^{k}\right)$ were chosen equal to 0.75 for calculation $\mathrm{A}$ and 0.70 for calculation $\mathrm{B}$, while the spin-orbit parameters were left at their ab initio values. These low values of the scaling factors have been suggested by Cowan for neutral heavy elements [30]. In Table 1, it is taken into account the configuration sets including core $[\mathrm{Xe}]$ for calculations $\mathrm{A}$ and $\mathrm{B}$ of La II. The energies and the Landé $g$-factors for $5 d^{2}, 5 d 6 s$, $6 s^{2}, 4 f 6 p, 5 d 7 s, 5 d 6 d, 4 f^{2}, 6 p^{2}, 6 s 6 d, 6 s 7 s, 4 f 6 s, 4 f 5 d, 5 d 6 p$, $6 \mathrm{~s} 6 \mathrm{p}, 4 \mathrm{f} 7 \mathrm{~s}, 4 \mathrm{f} 6 \mathrm{~d}, 5 \mathrm{~d} 7 \mathrm{p}$, and $6 \mathrm{~s} 7 \mathrm{p}$ excited levels are presented in Table 2. The agreement between our energies and the Landé $g$-factors from the HFR calculation obtained according to A and $\mathrm{B}$ configuration sets and other works is very good.

These energy data and the Landé $g$-factors for La II can be useful in investigations of some radiative properties and interpretation of many levels of La II. Because magnetic fields play a major role in many scientific areas such as astrophysics, the calculation of the Landé $g$-factor is important. The experimental values for Landé $g$-factors of rare-earth elements are far from being complete. We hope that the results reported in this work will be useful in the interpretation of atomic spectra of La II. Many feature characteristics of the spectra of neutral atom or ions of lanthanides remain preserved for lanthanide ions implemented in crystals. This is one reason for the wide interest in the application of lanthanides as active media in lasers. In addition, knowledge of electronic levels of lanthanides is important in astrophysics, since it allows precise determination of the abundance of particular elements. Also, the analysis of electronic levels is very important for a description of the interaction in creating chemical bonds or crystalline lattice. Consequently, we hope that our results obtained using the HFR and MCHF methods will be useful for other works in the future for La II spectra.

\section{References}

[1] B. Furmann, J. Ruczkowski, D. Stefańska, M. Elantkowska, and J. Dembczyński, "Hyperfine structure in La II odd configuration levels," Journal of Physics B, vol. 41, no. 21, Article ID 215004, 8 pages, 2008.

[2] J. E. Lawler, G. Bonvallet, and C. Sneden, "Experimental radiative lifetimes, branching fractions, and oscillator strengths for La II and a new determination of the solar lanthanum abundance," The Astrophysical Journal, vol. 556, no. 1, pp. 452-460, 2001.
[3] W. F. Meggers, "The structure of the La II spectrum," Journal of the Optical Society of America, vol. 14, no. 3, pp. 191-204, 1927.

[4] W. F. Meggers, "The Strongest lines of singly ionized atoms," Journal of the Optical Society of America, vol. 31, no. 10, pp. 605611, 1941.

[5] H. N. Russell and W. F. Meggers, "An analysis of lanthanum spectra (La I, La II, La III)," Journal of Research of the National Bureau of Standards, vol. 9, pp. 625-628, 1932.

[6] N. Grevesse and G. Blanquet, "Abundances of the rare earths in the sun," Solar Physics, vol. 8, no. 1, pp. 5-17, 1969.

[7] N. Spector and U. Gotthelf, "Configuration interaction in singly ionized lanthanum (La II)," Óptica Pura Y Aplicada, vol. 3, pp. 98-103, 1970.

[8] X. P. Xie, C. B. Xu, W. Sun et al., "Study of $\mathrm{La}^{+}$Rydberg and autoionization states: ionization potential of La II," Journal of the Optical Society of America B, vol. 16, no. 3, pp. 484-487, 1999.

[9] E. F. Worden, R. W. Solarz, J. A. Paisner, and J. G. Conway, "First ionization potentials of lanthanides by laser spectroscopy," Journal of the Optical Society of America, vol. 68, no. 1, pp. 52-61, 1978.

[10] J. Sugar and J. Reader, "Ionization energies of the singly ionized rare earths," Journal of the Optical Society of America, vol. 55, no. 10, pp. 1286-1290, 1965.

[11] E. Eliav, S. Shmulyian, U. Kaldor, and Y. Ishikawa, "Transition energies of lanthanum, actinium, and eka-actinium (element 121)," Journal of Chemical Physics, vol. 109, no. 10, pp. 3954-3958, 1998.

[12] D. Kułaga-Egger and J. Migdałek, "Theoretical radiative lifetimes of levels in singly ionized lanthanum," Journal of Physics B, vol. 42, no. 18, Article ID 185002, 6 pages, 2009.

[13] D. J. Bord, L. P. Barisciano, and C. R. Cowley, "Gf-values for singly ionized lanthanum based on a new calibration of NBS Monograph 145 intensities," Monthly Notices of the Royal Astronomical Society, vol. 278, no. 4, pp. 997-1004, 1996.

[14] T. Andersen, O. Poulsen, P. S. Ramanujam, and A. P. Petkov, "Lifetimes of some excited states in the rare earths: La II, Ce II, Pr II, Nd II, Sm II, Yb I, Yb II, and Lu II," Solar Physics, vol. 44, no. 2, pp. 257-267, 1975.

[15] A. Arnesen, A. Bengtsson, R. Hallin, J. Lindslog, C. Nordling, and T. Noreland, "Lifetime measurements in La II with the beam-laser method," Physica Scripta, vol. 16, no. 1-2, pp. 31-34, 1977.

[16] A. Arnesen, A. Bengtsson, R. Hallin, and T. Noreland, "Lifetime measurements the La II $\mathrm{y}_{3} \mathrm{~F}_{4,3,2}^{0}$ levels with the beam-laser method (solar photosphere la abundance)," Journal of Physics $B$, vol. 10, no. 4, article 010, pp. 565-568, 1977.

[17] Z. S. Li and Z.-K. Jiang, "Lifetime measurements in La II and La III using time-resolved laser spectroscopy," Physica Scripta, vol. 60 , no. 5, pp. 414-417, 1999.

[18] J. Migdalek and W. E. Baylis, "Multiconfiguration Dirac-Fock study of the $5 \mathrm{~d} 6 \mathrm{p}^{3} \mathrm{~F}_{4}^{\circ}$ lifetime in singly ionized lanthanum," Physical Review A, vol. 43, no. 9, pp. 4625-4628, 1991.

[19] Z. Zhiguo, L. Zhongshan, and J. Zhankui, "Experimental investigations of oscillator strengths for ultraviolet transitions in La II," European Physical Journal D, vol. 77, no. 4, pp. 499-502, 1999.

[20] A. Derkatch, L. Ilyinsky, S. Mannervik et al., "Experimental and theoretical investigation of radiative decay rates of metastable levels in La II," Physical Review A, vol. 65, no. 6, pp. 625081625087, 2002.

[21] C. Höhle, H. Hühnermann, and H. Wagner, "Measurements of the hyperfine structure constants of all the $5 d^{2}$ and $5 d 6 s$ levels 
in ${ }^{139} \mathrm{La}$ II using the high-resolution spectroscopy on collinear laser-ion-beams," Zeitschrift Für Physik A Hadrons and Nuclei, vol. 304, no. 4, pp. 279-283, 1982.

[22] J. Bauche, J.-F. Wyart, Z. Ben Ahmed, and K. Guidara, "Interpretation of the hyperfine structures in the low even configurations of lanthanum II," Zeitschrift für Physik A Atoms and Nuclei, vol. 304, no. 4, pp. 285-292, 1982.

[23] L. Maosheng, M. Hongliang, C. Miaohua et al., "Hyperfine structure measurements in the lines $576.91 \mathrm{~nm} 597.11 \mathrm{~nm}$ and $612.61 \mathrm{~nm}$ of La 1l," Physica Scripta, vol. 61, no. 4, pp. 449-451, 2000.

[24] H. Iimura, M. Koizumi, M. Miyabe et al., "Nuclear moments and isotope shifts of ${ }^{135} \mathrm{La},{ }^{137} \mathrm{La}$, and ${ }^{138} \mathrm{La}$ by collinear laser spectroscopy," Physical Review C, vol. 68, Article ID 054328, 5 pages, 2003.

[25] P. Schef, M. Björkhage, P. Lundin, and S. Mannervik, "Precise hyperfine structure measurements of La II utilizing the laser and rf double resonance technique," Physica Scripta, vol. 73, no. 2, pp. 217-222, 2006.

[26] D. Datta and D. R. Beck, "Relativistic many-body effects in the fine and hyperfine structure of ${ }^{139} \mathrm{La}$ II, $(5 d+6 s)^{2} J=2$ states: the need for second-order electrostatic corrections," Physical Review A, vol. 52, no. 5, pp. 3622-3627, 1995.

[27] J. E. Sansonetti and W. C. Martin, "Handbook of basic atomic spectroscopic data," Journal of Physical and Chemical Reference Data, vol. 34, pp. 1559-2259, 2005.

[28] http://www.nist.gov/physlab/data/asd.cfm.

[29] C. F. Fischer, T. Brage, and P. Jönsson, Computational Atomic Structure-An MCHF Approach, IOP, Bristol, England, 1997.

[30] R. D. Cowan, The Theory of Atomic Structure Spectra, University of California Press, California, Calif, USA, 1981.

[31] B. Karaçoban and L. Özdemir, "Energies and lifetimes for some excited levels in La I," Acta Physica Polonica A, vol. 113, no. 6, pp. 1609-1618, 2008.

[32] B. Karaçoban and L. Özdemir, "Electric dipole transitions for La I, $(Z=57)$," Journal of Quantitative Spectroscopy and Radiative Transfer, vol. 109, no. 11, pp. 1968-1985, 2008.

[33] B. Karaçoban and L. Özdemir, "The hyperfine structure calculations of some excited levels for ${ }^{139}$ La I," Acta Physica Polonica A, vol. 115, no. 5, pp. 864-872, 2009.

[34] B. Karaçoban and L. Özdemir, "Transition energies of neutral and singly ionized lanthanum," Indian Journal of Physics, vol. 84, no. 3, pp. 223-230, 2010.

[35] B. Karaçoban and L. Özdemir, "Electric dipole transitions for Lu I, $(Z=71)$," Arabian Journal for Science and Engineering, vol. 36, no. 4, pp. 635-648, 2011.

[36] B. Karaçoban and L. Özdemir, "Energies and Landé factors for some excited levels in Lu I, $(Z=71)$," Central European Journal of Physics, vol. 9, no. 3, pp. 800-806, 2011.

[37] B. Karaçoban and L. Özdemir, "Energies, Landé factors, and lifetimes for some excited levels of neutral ytterbium $(Z=70)$," Acta Physica Polonica A, vol. 119, no. 3, pp. 342-353, 2011.

[38] B. Karaçoban and L. Özdemir, "Electric dipole transitions for neutral ytterbium $(Z=70)$," Journal of the Korean Physical Society, vol. 58, no. 3, pp. 417-428, 2011.

[39] B. Karaçoban and L. Özdemir, "Transition energies of ytterbium $(Z=70)$," Zeitschrift Für Naturforschung A, vol. 66, pp. 543-551, 2011.

[40] B. Karaçoban and L. Özdemir, "The level structure of atomic lutetium $(Z=71)$ : a relativistic Hartree-Fock calculation," Indian Journal of Physics, vol. 85, no. 5, pp. 683-702, 2011.
[41] B. Karaçoban and L. Özdemir, "Transition energies of lutetium," Chinese Journal of Physics, vol. 50, no. 1, pp. 40-49, 2012.

[42] B. Karaçoban and L. Özdemir, "Transition parameters for doubly ionized lanthanum," Journal of Atomic, Molecular, and Optical Physics, vol. 2012, Article ID 246105, 15 pages, 2012.

[43] P. Jönsson and S. Gustafsson, "A program for computing weak and intermediate field Zeeman splittings from MCHF wave functions," Computer Physics Communications, vol. 144, no. 2, pp. 188-199, 2002.

[44] C. F. Fischer, "The MCHF atomic-structure package," Computer Physics Communications, vol. 128, no. 3, pp. 635-636, 2000.

[45] http://www.tcd.ie/Physics/People/Cormac.McGuinness/Cowan/. 

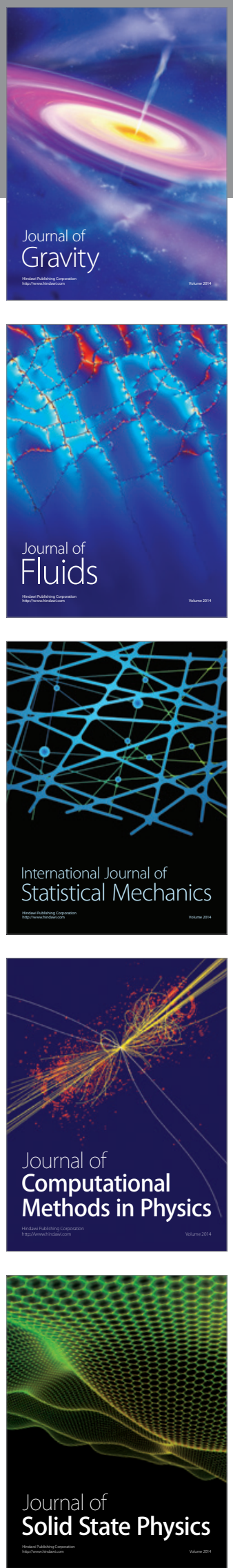

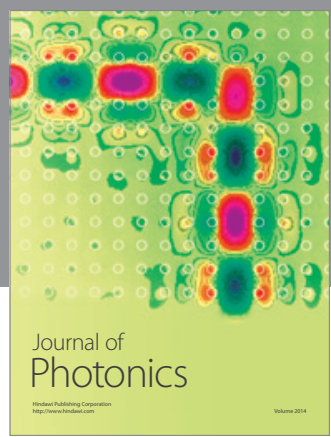

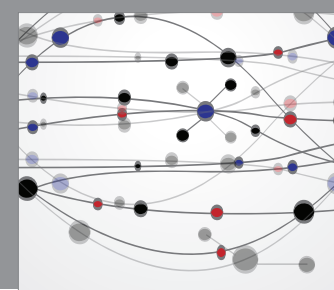

The Scientific World Journal

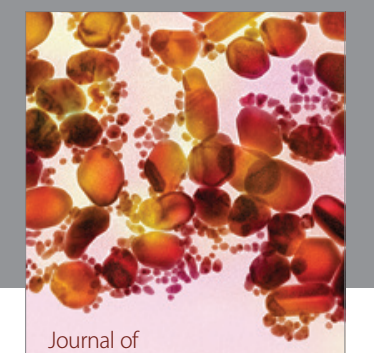

Soft Matter
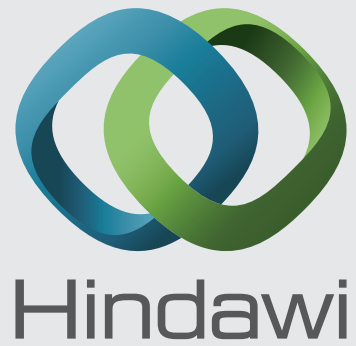

Submit your manuscripts at

http://www.hindawi.com
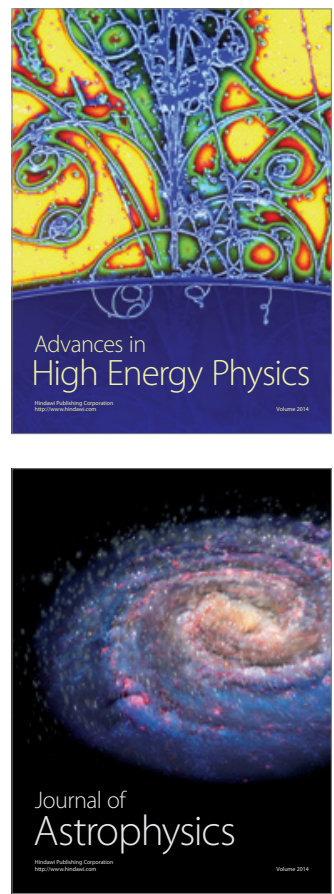
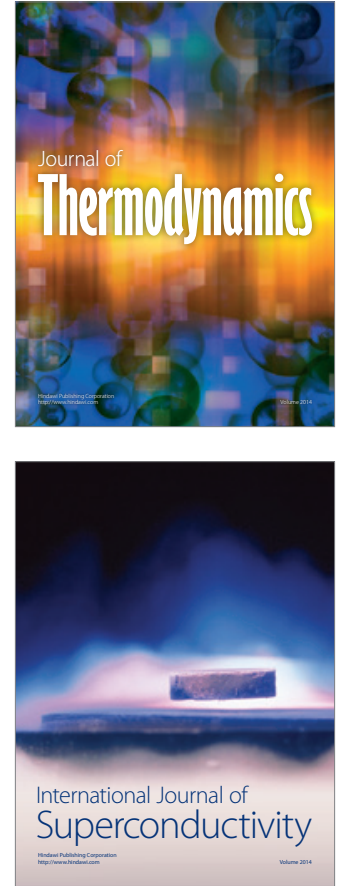
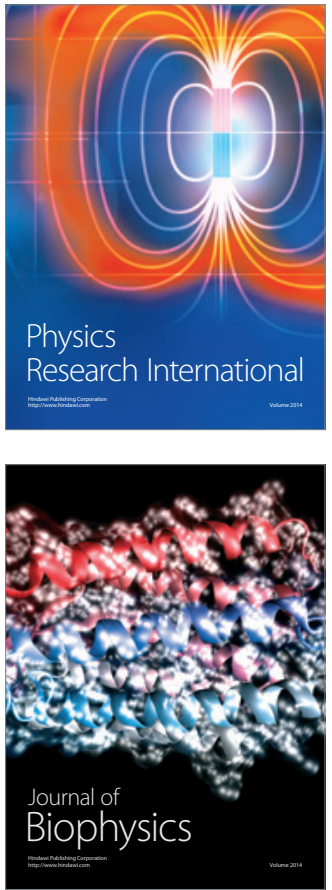
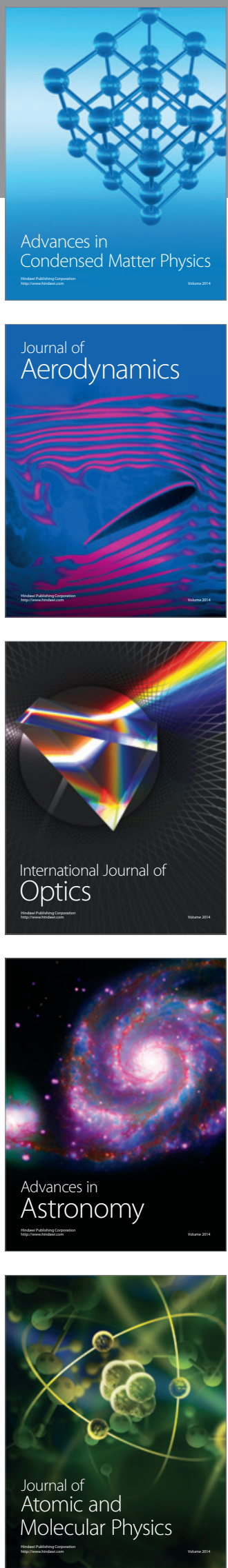